OPEN ACCESS

Edited by:

Bryon Johnson,

Medical College of Wisconsin,

United States

Reviewed by:

Sabrin Albeituni,

St. Jude Children's Research Hospital,

United States

Edmund Waller.

Emory University, United States

*Correspondence:

Luigi Racioppi

luigi.racioppi@duke.edu

Nelson Chao

chao0002@mc.duke.edu

Specialty section:

This article was submitted to

Cancer Immunity

and Immunotherapy,

a section of the journal

Frontiers in Immunology

Received: 05 August 2021 Accepted: 27 September 2021

Published: 12 October 2021

Citation:

Huang W, Liu Y, Luz A, Berrong M,

Meyer JN, Zou Y, Swann E,

Sundaramoorthy $P$, Kang $Y$, Jauhari $S$,

Lento W, Chao N and Racioppi L

(2021) Calcium/Calmodulin

Dependent Protein Kinase

Kinase 2 Regulates the Expansion of Tumor-Induced Myeloid-Derived

Suppressor Cells.

Front. Immunol. 12:754083. doi: 10.3389/fimmu.2021.754083

\section{Calcium/Calmodulin Dependent Protein Kinase Kinase 2 Regulates the Expansion of Tumor-Induced Myeloid-Derived Suppressor Cells}

\author{
Wei Huang ${ }^{1}$, Yaping Liu ${ }^{1}$, Anthony Luz ${ }^{2}$, Mark Berrong ${ }^{3}$, Joel N. Meyer ${ }^{2}$, Yujing Zou ${ }^{1}$, \\ Excel Swann ${ }^{1}$, Pasupathi Sundaramoorthy ${ }^{1}$, Yubin Kang ${ }^{1}$, Shekeab Jauhari ${ }^{1}$, \\ William Lento ${ }^{1}$, Nelson Chao ${ }^{1 *}$ and Luigi Racioppi ${ }^{1,4^{*}}$ \\ ${ }^{1}$ Division of Hematological Malignancies and Cellular Therapy, Department of Medicine, Duke University Medical Center, \\ Durham, NC, United States, ${ }^{2}$ Duke University Nicholas School of the Environment, Durham, NC, United States, ${ }^{3}$ Duke \\ Human Vaccine Institute, Durham, NC, United States, ${ }^{4}$ Department of Molecular Medicine and Medical Biotechnology, \\ University of Naples Federico II, Naples, Italy
}

Myeloid-derived suppressor cells (MDSCs) are a hetero geneous group of cells, which can suppress the immune response, promote tumor progression and impair the efficacy of immunotherapies. Consequently, the pharmacological targeting of MDSC is emerging as a new immunotherapeutic strategy to stimulate the natural anti-tumor immune response and potentiate the efficacy of immunotherapies. Herein, we leveraged genetically modified models and a small molecule inhibitor to validate Calcium-Calmodulin Kinase Kinase 2 (CaMKK2) as a druggable target to control MDSC accumulation in tumor-bearing mice. The results indicated that deletion of CaMKK2 in the host attenuated the growth of engrafted tumor cells, and this phenomenon was associated with increased antitumor $T$ cell response and decreased accumulation of MDSC. The adoptive transfer of MDSC was sufficient to restore the ability of the tumor to grow in Camk k2/- mice, confirming the key role of MDSC in the mechanism of tumor rejection. In vitro studies indicated that blocking of CaMKK2 is sufficient to impair the yield of MDSC. Surprisingly, MDSC generated from Camkk2 ${ }^{-/}$bone marrow cells also showed a higher ability to terminally differentiate toward more immunogenic cell types (e.g inflammatory macrophages and dendritic cells) compared to wild type (WT). Higher intracellular levels of reactive oxygen species (ROS) accumulated in Camkk2 $2^{-/}$MDSC, increasing their susceptibility to apoptosis and promoting their terminal differentiation toward more mature myeloid cells. Mechanistic studies indicated that AMP-activated protein kinase (AMPK), which is a known CaMKK2 proximal target controlling the oxidative stress response, fine-tunes ROS accumulation in MDSC. Accordingly, failure to activate the CaMKK2-AMPK axis can account for the elevated ROS levels in Camkk2 ${ }^{-/-}$MDSC. These results highlight CaMKK2 as an important regulator of the MDSC lifecycle, identifying this kinase as a new druggable target to restrain MDSC expansion and enhance the efficacy of anti-tumor immunotherapy.

Keywords: tumor microenvionment, MDSC (myeloid-derived suppressor cell), anti-tumor immune response, CaMKK $\beta$, AMPK, ROS - reactive oxygen species 


\section{INTRODUCTION}

Immunosuppressive myeloid cells showing the phenotype of neutrophils and monocytes were originally described more than 20 years ago and later, these cells were named myeloidderived suppressor cells (MDSCs) (1). For many years the features that distinguish MDSCs from conventional neutrophils and monocytes have been poorly understood. However, leveraging genomic, proteomic, and metabolic approaches, emerging studies have contributed to the identification of features that distinguish MDSCs from conventional neutrophils and monocytes $(2,3)$. In mice, MDSCs are phenotypically identified as $\mathrm{CD} 11 \mathrm{~b}^{+} \mathrm{Gr} 1^{+}$cells and are further categorized according to $\mathrm{Gr}-1$ subgroup markers as either monocytic $\left(\mathrm{CD} 11 \mathrm{~b}+\mathrm{Ly} 6 \mathrm{C}^{\text {high }}\right.$ Ly6 $\left.\mathrm{G}^{\text {neg }}, \mathrm{M}-\mathrm{MDSC}\right)$ or granulocytic MDSC (CD11b+Ly6C ${ }^{\text {low }}$ Ly6 $6 \mathrm{G}^{\text {high }}$, G-MDSC) (2, $4,5)$. In humans, M- and G-MDSCs are defined as CD11b+ CD14+CD15- and CD11b+CD14-CD15+, respectively. MDSCs suppress the immune response through multiple mechanisms including the release of reactive oxygen species (ROS), nitric oxide (NO), indoleamine 2,3-dioxygenase, and suppressive cytokines (2, 6-9). MDSCs also have the ability to deplete tissues of arginine required for $\mathrm{T}$-cell proliferation. The development of MDSCs seems to involve two phases that ultimately lead to the accumulation of pathologically activated MDSCs into the tumor microenvironment (TME) (10). During the initial phases, the tumor-derived signals drive the expansion and conditioning of bone marrow and spleen resident myeloid progenitors toward MDSCs. Subsequently, MDSCs are recruited and pathologically activated in the TME, where M-MDSC terminally differentiate into tumor-associated macrophages (2). Overall, MDSCs function to facilitate the escape of tumor cells from the immune surveillance and the accumulation of these cell types in TME correlates with tumor stage, progression, and resistance to standard chemotherapy, radiotherapy, and immunotherapy (11-14). For these reasons, the druggable components of the molecular machinery that controls MDSC lifecycle are becoming attractive immunotherapeutic targets to re-shape the immunosuppressive TME and enhance the efficacy of immunotherapies (15).

One such potential pathway is calcium signaling. Calcium ions $\left(\mathrm{Ca}^{2+}\right)$ are an important and pervasive second messenger that regulates a plethora of cell functions (16). Most of the effects of $\mathrm{Ca}^{2+}$ are mediated by Calmodulin (CaM), which is a ubiquitously expressed small protein that functions as the primary intercellular $\mathrm{Ca}^{2+}$ sensor. Once formed, the $\mathrm{Ca}^{2+} / \mathrm{CaM}$ complexes can bind and regulate the activity of Calcium/ Calmodulin Kinase proteins (CaMKs), which is a family of Ser/Thr kinases including $\mathrm{Ca}^{2+} \mathrm{CaM}$-dependent protein kinase I (CaMKI), CaMKIV, $\mathrm{Ca}^{2+1} \mathrm{CaM}$-dependent protein kinase kinase 1 (CaMKK1) and CaMKK2 (17). Following activation by $\mathrm{Ca}^{2+} / \mathrm{CaM}$ complexes, CaMKK1 and 2 can further phosphorylate and stimulate CaMKI and CaMKIV kinase activity. Importantly, only CAMKK2 can phosphorylate and activate 5' AMP-activated kinase (AMPK) $(18,19)$, which has an important role in the control of cellular energy metabolism, oxidative stress response, and processes that regulate innate and adaptive immunity $(20,21)$. Outside the brain, CaMKK2 is expressed at detectable levels restricted to a small subset of cell types, including hematopoietic stem and progenitor cells (HSCP) and mature myeloid cell subsets such as circulating monocytes and macrophages (22). CaMKK2 also regulates granulopoiesis and recently we have demonstrated that this kinase controls HSCP regeneration following bone marrow injury $(23,24)$. Interestingly, CaMKK2 impinges the activation program of macrophages, and consequently, genetic ablation of Camkk2 attenuates the detrimental inflammatory response induced by bacterial endotoxin or obesity (25). More recently, we demonstrated that CaMKK2 is a key regulator of the immune-suppressive microenvironment in breast cancer and blockade of this enzyme attenuates tumor growth in a CD8+ T cell-dependent manner (26). Hence, CaMKK2 is an important druggable target of therapies aimed to counteract tumorinduced immunosuppression and foster the anti-tumor immune response.

In this study, we investigated the role of CaMKK2 in MDSCs. To this end, we leveraged germline and inducible models of Camkk2-deficient mice in combination with in vivo and in vitro models of MDSC expansion. The results from these studies indicated that deletion of CaMKK2 in the host attenuated the growth of engrafted tumor cells, and this phenomenon was associated with increased antitumor $\mathrm{T}$ cells response and decreased accumulation of MDSCs. Mechanistically we show that the CaMKK2-AMPK axis controls the survival and the terminal differentiation of tumor-induced MDSCs by finetuning the accumulation of reactive oxygen species (ROS). Translationally, these results identify CaMKK2 as a novel druggable target to restrain MDSC expansion and enhance the efficacy of anti-tumor immunotherapy.

\section{MATERIALS AND METHODS}

\section{Mice}

C57BL/6 mice were purchased from the Jackson Laboratory (Jackson Laboratory, CA, USA). CaMKK2 global knock-out $\left(C_{\left.a m k k 2^{-/}\right)}\right)$Tg (Camkk2-EGFP)DF129Gsat mice were generated as described $(27,28)$ and backcrossed to C57BL/6 for at least 8 generations. LysMCre ${ }^{+} ; \mathrm{CaMKK}^{\mathrm{fl} / \mathrm{fl}}$ and littermates control were generated by crossing B6.129P2-Lyz2tm1(cre)Ifo/J mice from the Jackson Laboratory with CaMKK2 $2^{\text {lox/lox }}$ mice (29). ERT2-Cre; CaMKK2 ${ }^{\mathrm{f} / \mathrm{ll}}$ were generated by crossing B6.Cg-Tg $\left(\mathrm{CAG}-\mathrm{cre} / \mathrm{Esr}^{*}{ }^{*}\right) 5 \mathrm{Amc} / \mathrm{J}$ transgenic mice from the Jackson laboratory with $\mathrm{CaMKK} 2^{\text {lox/lox }}$ mice (29). All the mice were used between 8-16 weeks of age and were gender and agematched. Animal care and experimental procedures were approved by the National Institute of Health and the Duke University Institutional Animal Care and Use Committee.

\section{E.G7-OVA Tumor Model}

E.G7-OVA cells were purchased from American Type Tissue Culture Collection (Rockville, MA, USA). For bioluminescent analysis, E.G7-OVA cells were stably transfected with a plasmid 
expressing luciferase. The cells were cultured in RPMI 1640 supplied with $4.5 \mathrm{~g} / \mathrm{L}$ glucose, $2 \mathrm{mM}$ L-glutamine, $1.5 \mathrm{~g} / \mathrm{L}$ sodium bicarbonate, 10mM HEPES, 1.0mM sodium pyruvate (all from Gibco, MA, USA), 0.05mM 2-mercaptoethanol, 0.4mg/ml G418 (Sigma-Aldrich, MO, USA), and $10 \%$ fetal bovine serum (Hyclone, MA, USA) in humidified $37^{\circ} \mathrm{C} \mathrm{CO}_{2}$ incubator. E.G7OVA cells were subcutaneously injected into the flank and tumors were monitored using weekly bioluminescence imaging. The tumors were measured with a calibrator every 3 4 days and the volume was calculated as Length $\times$ Width $\times$ Width/2. EL4 T lymphoma cells (ATCC TIB-39) were purchased from Duke Cell Culture Facility and were cultured in RPMI 1640 supplied with $4.5 \mathrm{~g} / \mathrm{L}$ glucose, $2 \mathrm{mM}$ L-glutamine, $1.5 \mathrm{~g} / \mathrm{L}$ sodium bicarbonate, 10mM HEPES, 1.0mM sodium pyruvate (all from Gibco, MA, USA), 0.05mM 2-mercaptoethanol, and 10\% fetal bovine serum (Hyclone, MA, USA) in humidified $37^{\circ} \mathrm{C} \mathrm{CO}_{2}$ incubator. EL4 tumors were measured with a calibrator every 3-4 days and the volume was calculated as described previously. EO771 (30), 4T1 (31), MET1 (32), and A7C11 (33) epithelial mammary tumor cell lines were cultured in RPMI 1640 supplied with $4.5 \mathrm{~g} / \mathrm{L}$ glucose, $2 \mathrm{mM}$ L-glutamine, $1.5 \mathrm{~g} / \mathrm{L}$ sodium bicarbonate, 10mM HEPES, 1.0mM sodium pyruvate (all from Gibco, MA, USA) and $8 \%$ of $10 \%$ fetal bovine serum (Hyclone, $\mathrm{MA}, \mathrm{USA}$ ) in humidified $37^{\circ} \mathrm{C} \mathrm{CO}_{2}$ incubator. To generate tumor conditioned medium (TCM), regular media from 70$80 \%$ confluent cell cultures were removed and replaced with fresh culture medium. After 72 hours TCMs were then harvested, aliquoted, and stored at $-80^{\circ} \mathrm{c}$ before use.

\section{MDSC Transfusion}

MDSCs were isolated from the spleens of WT and Camkk2 $2^{-/}$ mice 21 days after E.G7-OVA injection and were enriched by anti-mouse Gr-1 Microbeads (Miltenyi, Bergisch Gladbach, Germany) according to the manufacturer's instructions. Then, $2 \times 10^{6}$ MDSCs (> 90\% pure) were injected into Camkk2 $2^{-/-}$mice through the tail vein, followed by $1 \times 10^{5}$ E.G7-OVA tumor cells injected subcutaneously into the flank. Tumor size was measured as described above.

\section{T-Cell Depletion Treatment}

$\mathrm{Camkk2}^{-/-}$mice were injected peritoneally with anti-mouse CD8 monoclonal antibody or its IgG isotype (BioXCell, NH, USA) $0.2 \mathrm{mg} 4$ days and 1 day before tumor inoculation, and every 3 days after EG7 tumor cell injection. $\mathrm{CD}^{+} \mathrm{T}$ cells depletion was monitored by flow cytometry.

\section{Flow Cytometry Analysis}

Single-cell suspensions of splenocytes were prepared as described (34). The removed tumors were dissected with a gentleMACS dissociator (Miltenyi, Bergisch Gladbach, Germany) and then treated with collagenase I and DNase (Roche, Basel, Switzerland) for $45 \mathrm{~min}$ at $37^{\circ} \mathrm{C}$. The cells were filtered and stained with antibodies. The following antibodies were used in this study: anti-mouse CD11c, anti-mouse/human CD11b, anti-mouse Ly6G, anti-mouse F4/80, anti-mouse CD69, anti-mouse CD8a, anti-mouse CD4 anti-mouse Ly6C, anti-mouse I-A/I-E, antimouse CD25, anti-mouse CD40, Fixable Viability Dye eFluor ${ }^{\circledR}$
450 (BioLegend, CA, USA). Cell apoptosis, CFSE, H2DCFDA, and MitoTracker Green staining were measured under the manufacturer protocols (Life Technologies, USA). BD FACS Canto flow cytometer (BD, NJ, USA) and FlowJo software were used for flow cytometry analysis (TreeStar, OR, USA). Antibodies used in this set of experiments are listed in Supplementary Table 1.

\section{In Vitro MDSC Generation}

MDSCs were generated as previously reported (35). Briefly, bone marrow nucleated cells were cultured in DMEM supplied with $2 \mathrm{mM}$ L-glutamine, $1.5 \mathrm{~g} / \mathrm{L}$ sodium bicarbonate, $10 \mathrm{mM}$ HEPES, $1.0 \mathrm{mM}$ sodium pyruvate (all from Gibco, MA, USA), 10\% fetal bovine serum (Hyclone, MA, USA), 40ng/ml rmGM-CSF and $40 \mathrm{ng} / \mathrm{ml} \mathrm{rmIL-6}$ (BioLegend), with or without 50\% E.G7-OVA supernatant. The floating cells were collected on day 4 and enriched by anti-mouse Gr-1 Microbeads (Miltenyi). The cells with purity $>90 \%$ were used. In some experiments, the cells were stained with anti-mouse Ly6C, anti-mouse Ly6G, and antimouse/human CD11b (Biolegend) and sorted with BD FACS Aria II cell sorter (BD).

\section{T-Cell Proliferation Assay}

MDSCs were prepared as described above. T cells were separated from spleens of C57BL/6 mice and enriched using the PanT Cell Isolation Kit II (Miltenyi). $2 \times 10^{5} \mathrm{~T}$ cells labeled with CFSE (MitoSciences, OR, USA) and $1 \times 10^{5}$ MDSC were seeded into 96-well plates (Corning, NY, USA). Mouse T-activator CD3/ CD28 beads (ThermoFisher Scientific, MA, USA) were added to the culture media. The cells were cultured in a humidified incubator with $5 \% \mathrm{CO}_{2}$ for $72 \mathrm{~h}$ and analyzed using a BD FACS Canto flow cytometer (BD), and FlowJo software (TreeStar, OR, USA).

\section{ELISpot Assay}

Single-cell suspensions were generated from the spleens of WT and $\mathrm{Camkk2}^{-/-}$mice 17 days after E.G7-OVA injection. Splenocytes $\left(2 \times 10^{5} /\right.$ well) were seeded into 96-well MultiScreen Immubilon-P Filtration Plates (Millipore, USA) coated with anti-mouse IFN- $\gamma$ antibody (Affymetric eBioscience, USA). OVA protein was added at $100 \mu \mathrm{g} / \mathrm{ml}$. Cells were cultured in a humidified incubator with $5 \% \mathrm{CO}_{2}$ for $48 \mathrm{~h}$. The reactions were processed according to the protocol instructions. CTLImmunoSpot ${ }^{\circledR}$ S6 FluoroSpot Line (Cellular Technology Ltd, $\mathrm{OH}, \mathrm{USA}$ ) and ImmunoSpot software (Cellular Technology Ltd, $\mathrm{OH}$, USA) was used for analysis.

\section{Immunoblot}

Immunoblots were prepared as published (24). The following primary antibodies were used in this study: purified mouse antiCaM kinase kinase (BD Biosciences, CA, USA), anti-phosphoAMPK alpha (T172 40H9) rabbit mAb, anti-AMPK alpha (F6) mouse mAb, anti-phopho-Stat3 (Tyr705 D3A7) rabbit mAb, anti-Stat3 (124H6) mouse $\mathrm{mAb}$, anti- $\beta$ actin mouse $\mathrm{mAb}$ (all from Cell Signaling, CA, USA). Secondary antibodies were antimouse IgG Alexa Fluor 680 (Invitrogen, CA, USA) or anti-rabbit IgG IRDye800 conjugated (Rockland Immunochemicals, PA, 
USA). The image was taken using Odyssey CLx (LI-COR, NE, USA) and analyzed using ImageStudio (LI-COR, NE, USA). Antibodies used in this set of experiments are listed in Supplementary Table 2.

\section{Real-Time Quantitative RT-PCR Assay}

RNA was extracted using the RNeasy Mini Kit (Qiagen, Hilden, Germany). Quantitative PCR was performed using iQ SYBR Green Supermix (Bio-Rad, CA, USA) with respective primers and cDNA. The assay was run on the CFX96 Real-Time System (Bio-Rad, CA, USA). The primer sequences are listed in Supplementary Table 3.

\section{Cell Metabolic Assay}

OCR and ECAR were measured with the Seahorse XF24 extracellular flux analyzer (Agilent Technologies. CA, USA). Cells were attached to culture plates using Cell-Tak (BD Bioscience). OCR and ECAR were measured in un-buffered DMEM supplied with $4.5 \mathrm{~g} / \mathrm{L} \mathrm{D}$-glucose and $10 \mathrm{mM} \mathrm{L-}$ glutamine (Gibco, MA, USA). OCR and ECAR values were normalized to cell numbers. Data were analyzed using Seahorse Wave (Agilent Technologies. CA, USA).

\section{Statistical Analysis}

All statistical analyses were performed using Prism GraphPad (GraphPad Software, CA, USA) and Excel (Microsoft, WA, USA). For survival studies, the log-rank Mantel-Cox test was used. For tumor size and bioluminescent measurement, student t-test and multi-way ANOVA tests were used. For flow cytometry, real-time quantitative RT-PCR assay, and cytokine detection analysis, a two-tail student $t$-test was used. The level of significance was set at $P<0.05$. Bar graphs represent mean \pm SEM.

\section{RESULTS}

\section{Deletion of Camkk2 in the Host Inhibits Lymphoma Cells Growth}

Outside the brain, we originally found CaMKK2 selectively expressed in myeloid cells and macrophages where this kinase regulates the inflammatory response triggered by bacterial endotoxin (25). More recently, we have demonstrated that CaMKK2 expressed in myeloid cells is an important regulator of the immunosuppressive breast cancer microenvironment and blockade of this kinase in the host cells inhibits tumor growth (26). Based on these results, we hypothesize that, besides breast cancer, CaMKK2 expressed in the host cells might have a more general function in the mechanism regulating the anti-tumor immune response. Consequently, we used E.G7-OVA tumor cells, a well-established derivative model of the EL-4 murine lymphoma cells, which has been modified to express an ectopic $\mathrm{T}$ cell-recognizable ovalbumin peptide (36). The E.G7-OVA cells were further engineered to express luciferin as a trackable marker so bioluminescent imaging was combined with caliper measurements to monitor tumor development. E.G7-OVA cells were thus injected subcutaneously (SC) into the right flank of wild type (WT) and Camkk2 $2^{-/-}$mice and tumors monitored by bioluminescence and caliper. In WT mice injected with $1 \times 10^{4}$ E.G7-OVA cells, the tumors were detectable by bioluminescence by day 7 and gradually increased over the following two weeks (Figure 1A and Supplementary Figure S1A). Although tumors successfully engrafted in $\mathrm{Camkk2}^{-/-}$mice, the bioluminescent signal gradually decreased after 21 days and eventually disappeared after 32 days. To further corroborate this finding, the experiment was replicated using an increased number of tumor cells $\left(1 \times 10^{5}\right)$. However, even in the conditions of increased tumor cell input, we found that deletion of CaMKK2 in the host cells effectively suppressed syngeneic tumor cell growth (Figure 1B).

We next examined the cell types expressing Camkk2 in the tumor microenvironment and spleen of lymphoma-bearing mice by engrafting E.G7-OVA cells into the flank of [Tg(Camkk2EGFP)C57BL/6J] Camkk2-reporter mice (27). After 3 weeks the tumors and spleens were removed and embedded in paraffin for histology or enzymatically digested to prepare a single-cell suspension. We then used immunostaining and flow cytometry analyses to examine immune cell subsets (37). There were a significant number of stromal cells associated with E.G7-OVA tumors expressing EGFP reporter protein (Supplementary Figure S1B). Flow cytometry analysis confirmed this result and showed robust Camkk2-reporter activity in tumorassociated $\mathrm{CD} 1 \mathrm{~b}^{+}$myeloid cells, but minimal activity in $\mathrm{CD}_{11 b^{-}}$non-myeloid cells (Supplementary Figure S1C, left

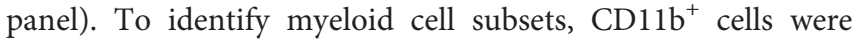
further sub-gated using Ly6C, Ly6G, F4/80, and MHC II markers (Supplementary Figures S1C, D). The Camkk2 promoter was highly active in the Ly6C $\mathrm{C}^{\text {high }} / \mathrm{Ly} 6 \mathrm{G}^{\mathrm{dim}}$ subset (M-MDSC) and $\mathrm{CD}_{11 \mathrm{~b}}{ }^{+}, \mathrm{Ly}_{6} \mathrm{C}^{\mathrm{dim}}$, Ly6G ${ }^{\mathrm{dim}}$, F4/80 ${ }^{+}$tumorassociated macrophage-like cells (Supplementary Figure S1C, lower left panel, DN gate). Conversely, we observed low levels of EGFP in the Ly6C $\mathrm{C}^{\mathrm{dim}} / \mathrm{Ly}_{6 \mathrm{G}} \mathrm{high}^{\text {sibset }}$ (G-MDSC) and Ly6C $\mathrm{C}^{\text {neg }} /$ $\mathrm{Ly}_{6 \mathrm{G}}{ }^{\mathrm{neg}} / \mathrm{F} 4 / 80^{\mathrm{dim} / \mathrm{neg}} / \mathrm{MHC}-\mathrm{II}^{\mathrm{dim} / \mathrm{neg}}$ cells (Supplementary Figure S1C). We next evaluated the expression of EGPF in the spleen from E.G7-OVA-bearing mice and control groups. MMDSC and DN splenocytes expressed the highest levels of EGFP reporter regardless of tumor status. However, the EGFP reporter was expressed at low levels in G-MDSC and barely detectable in $\mathrm{CD}_{11 b^{-}}$cells (Supplementary Figure S1D). These results indicate the Camkk2 promoter is highly active in tumorassociated myeloid cells and in myeloid subsets in the spleen of tumor-bearing mice.

We originally reported that CaMKK2 is significantly expressed in macrophages associated with the breast cancer microenvironment (26). Here we extend this finding to the lymphoma microenvironment, and more importantly, we identified spleen and tumor residing MDSCs as relevant cell types expressing this kinase. To determine the functional relevance of CaMKK2 expressed in myeloid cells in our model of hematological malignancy, we used LyMCre ${ }^{+}$Camkk $2^{\text {loxP }}$ mice in which Camkk2 is selectively deleted in the myeloid

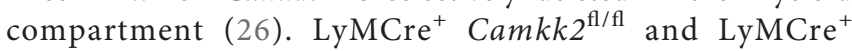


A

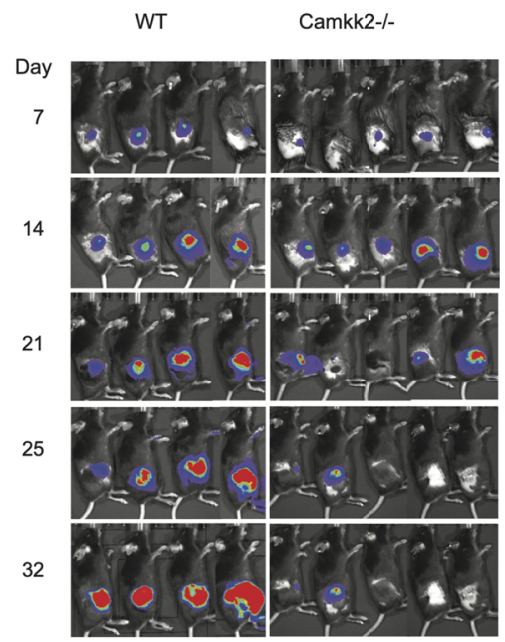

D

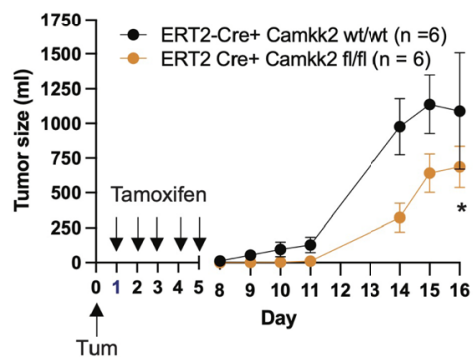

F

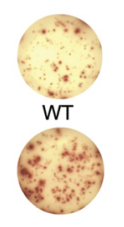

Camkk2-I-

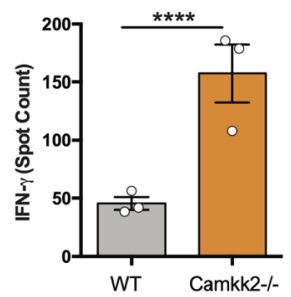

B

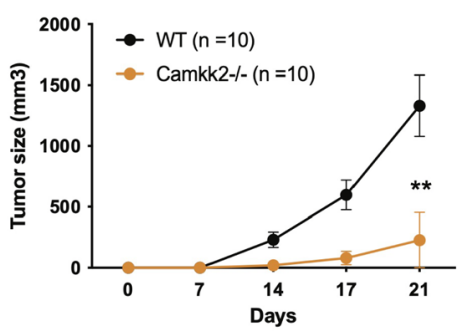

C

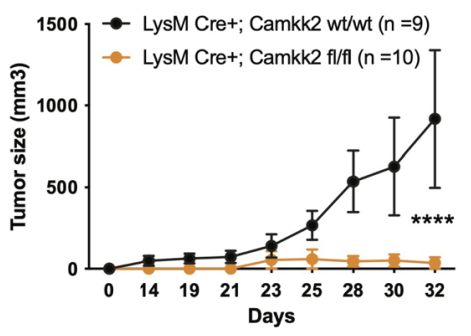

E

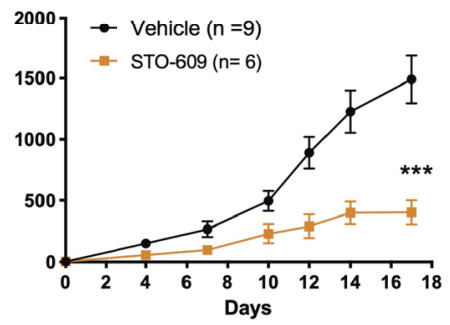

G

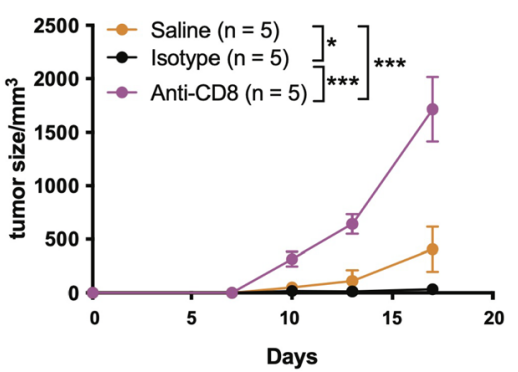

FIGURE 1 | Deletion of Camkk2 in the host inhibits lymphoma cells growth. WT and Camkk2 ${ }^{-1-}$ mice were injected with E.G7-OVA s.c. in the flank. The tumors were

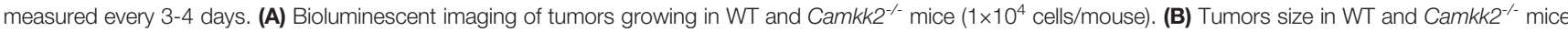
( $1 \times 10^{5}$ cells/mouse). $\mathrm{N}=10$ mice/group. Repeated twice. (C) Tumors size in LysM-Cre+; CaMKK2 ${ }^{\text {wt/wt }}$ and LysM-Cre+; CaMKK2 $2^{\mathrm{fl} / \mathrm{fl}} \mathrm{mice}\left(5 \times 10^{5} \mathrm{cellls} / \mathrm{mouse}^{\mathrm{m}}\right) . \mathrm{N}=$ 9 LysM-Cre+; CaMKK2 ${ }^{\text {wt/wt }}$ and 10 LysM-Cre+; CaMKK2 $2^{\text {ffllt }}$ mice. Combined from two experiments. (D) ERT2-Cre Camkk2 $2^{\text {loxp }}$ mice were injected with EL4 cells s.c. in the flank and treated with tamoxifen $(20 \mathrm{mg} / \mathrm{kg}$, daily, oral gavage). Tumor growth was monitored by caliper. (E) WT mice were injected with EL4 cells s.c. in the flank and treated with STO-609 (40 $\mu \mathrm{mol} / \mathrm{kg}$, IP at 48 hours interval, total 7-doses). (F) WT and Camkk2 $2^{-/}$mice were injected with $1 \times 10^{5}$ E.G7-OVA s.c. in the flank. After 17 days the spleens were removed, and anti-OVA T-cell response was assessed in splenocytes. N=3, repeated twice. (G) E.G7-OVA tumor growth in Camkk2 ${ }^{-1}$ mice treated with saline, anti-CD8+ or isotype control antibodies. N=4-5. Repeated twice. ${ }^{*},{ }^{* *},{ }^{\star * \star}$ and ${ }^{* \star \star *}$ refer to $p<0.05,0.01,0.005$, and 0.001 respectively.

Camkk2 $2^{\mathrm{wt} / \mathrm{wt}}$ littermates were thus challenged with E.G7-OVA cells subcutaneously. Consistent with our observations in the Camkk2 $2^{-/}$global knockout model, the E.G7-OVA tumors were significantly smaller in mice genetically deleted of Camkk2 in the myeloid cells compared to control littermates (Figure 1C). To determine the potential translational implications of this finding, we next leveraged our model of inducible Camkk2 knockout mice. We first demonstrated that treatment with tamoxifen was able to induce a significant downregulation of Camkk2 mRNA expression in the relevant myeloid cell population. Mice were treated with tamoxifen and then Camkk2 RNA expression was determined by QT-PCR in peritoneal lavage macrophages 
isolated from ERT2-Cre ${ }^{+} \mathrm{Camkk}_{2}^{\mathrm{fl} / \mathrm{fl}}$ and ERT2-Cre ${ }^{+} \mathrm{Camkk}^{\mathrm{fl} / \mathrm{fl}}$. The results from this experiment indicate that, following tamoxifen treatment, the expression of Camkk2 RNA was significantly lower in ERT2-Cre ${ }^{+} \mathrm{Camkk}^{\mathrm{fl} / \mathrm{fl}}$ compared to control littermates (Supplementary Figure S1E). EL-4 cells were next SC inoculated in ERT2-Cre ${ }^{+} \mathrm{Camkk}^{\mathrm{fl} / \mathrm{fl}}$ mice and their control littermates (ERT2-Cre ${ }^{+} \mathrm{Camkk}^{\mathrm{wt} / \mathrm{wt}}$ ) and mice were then treated with tamoxifen. The results of these studies indicated that the acute deletion of Camkk2 in the host cells was sufficient to induce significant inhibition of the lymphoma cells growth (Figure 1D). Lastly, we evaluated the efficacy of STO-609, a small molecule pharmacological CaMKK2 inhibitor (38), to suppress the growth of EL-4 lymphoma cells. Mice were injected with EL-4 cells and 24 hours later were treated with STO-609 for 2 weeks. Similar to findings observed in genetically modified models of Camkk2 deletion (Figures 1A-D), tumors growing in mice treated with STO-609 were significantly smaller compared to mice receiving vehicle alone (Figure 1E). Although this pharmacological approach cannot distinguish the intrinsic effect of STO-609 on tumor cells from those on stroma cells, it provides further evidence on the potential translational impact of targeting CaMKK2 to restrain lymphoma growth.

\section{Deletion of Camkk2 in the Host Enhances Anti-Tumor T-Cell Mediated Immunity}

$\mathrm{CD}^{+} \mathrm{T}$ cells have an important role in the mechanism of tumor rejection and consequently, an increased number of $\mathrm{CD}^{+}$ tumor-infiltrating lymphocytes (TIL) has been associated with a good prognosis in several tumor types (39). Moreover, we have previously shown that deletion of CaMKK2 in the host restrains mammary cancer growth, and this phenomenon was associated with increased accumulation of $\mathrm{T}$ cells in the tumor microenvironment and prevented by depletion of $\mathrm{CD}^{+} \mathrm{T}$ cells (26). To determine whether a similar mechanism is also present in the lymphoma tumor model, we first analyzed the percentage and phenotype of TILs in EG-7-OVA tumors in WT and $\mathrm{Camkk2}^{-/-}$mice. To this end, E.G7-OVA cells were injected into the flank and the TIL phenotype was then analyzed by FACS in tumors removed 14-17 days later. The data indicated comparable percentages of both $\mathrm{CD} 4^{+}$and $\mathrm{CD} 8^{+}$ $\mathrm{T}$ cells in tumors removed from $\mathrm{Camkk}^{-/-}$and WT mice (Supplementary Figures S2A, B). However, the percentage of CD8+ GranzymeB ${ }^{+}$PD1 $^{-}$TIL, a phenotype associated with non-exhausted effector $\mathrm{T}$ cells, was increased in E.G7OVA tumor removed from Camkk2 $2^{-/}$compared to WT mice (Supplementary Figure S2B). Of note, changes in the TIL repertoire observed in lymphoma models resemble those originally described in mammary tumors growing in Camkk2 $2^{-/-}$ compared to WT mice (26), indicating that in these two models of tumors deletion of Camkk2 in the host trigger a similar antitumor effector mechanism.

We next leveraged the EG7-OVA lymphoma model to determine the ability of WT and Camkk2 $2^{-/}$mice to develop a specific $\mathrm{T}$ cell-mediated response toward the OVA antigen, which is selectively expressed by E.G7-OVA lymphoma cells. Fresh splenocytes were isolated from E.G7-OVA-bearing mice and then cultured in vitro in the presence of OVA protein. The cells were then analyzed for IFN- $\gamma$ production by ELISpot. We found three-fold more IFN- $\gamma$ spots in splenocytes isolated from Camkk2 ${ }^{-/}$compared to WT mice, which suggests the decrease of MDSC in $\mathrm{Camkk2}^{-/-}$mice unleashes an anti-tumor immune response that restrains tumor growth (Figure 1F). Lastly, we determined the role of CD8+ T-cell-mediated immune response in the lymphoma growth inhibition mechanism. To this end, Camkk2 $2^{-/-}$mice were treated with anti-CD8 depleting antibody or isotype control and subsequently challenged with E.G7-OVA cells (Supplementary Figure S2C). Tumor growth was suppressed in $\mathrm{Camkk2}^{-/-}$mice that received no treatment or isotype IgG control, while the treatment with anti-CD8 depleting antibody reversed the protective effects of Camkk2 deletion, enabling the growth of E.G7-OVA (Figure 1G). These findings indicated that the loss of CaMKK2 in the host cells enhances the anti-tumor $\mathrm{T}$ cell response, and in turn suppressess lymphoma cells to grow in Camkk2 $2^{-/-}$mice.

\section{Deletion of Camkk2 in the Host Prevents Tumor-Induced MDSC Expansion}

The accumulation of MDSCs in lymphoid tissues and blood of tumor-bearing individuals is an important immunosuppressive phenomenon associated with poor prognosis in preclinical tumor models and humans (5). We thus hypothesized that CaMKK2 regulates myeloid cell responsiveness to tumorderived factors, and inhibition of this kinase in host cells prevents the tumor-induced MDSC expansion. To test this hypothesis, we determined the effect of Camkk2 deletion on MDSCs accumulating in the spleen of normal and tumorbearing mice. In normal conditions (CTR), WT and Camkk2 ${ }^{-1-}$ mice had comparable CD $11 \mathrm{~b}^{+}$and Ly6C $\mathrm{C}^{\text {high }}$ Ly6G ${ }^{\text {dim }}$ percentage in the spleens, while Camkk2 $2^{-/}$mice had a slight increase of Ly6G ${ }^{\text {dim }}$ Ly6G ${ }^{\text {high }}$ compared to WT (Figures 2A, B). Regardless of the genotype, a significant increase in the percentage of MDSCs was detected in EG7-OVA-bearing (TB) mice compared to the control groups (Figure 2B). Interestingly, less MDSC accumulated in the spleens and in the tumors of Camkk2 $2^{-/}$compared to WT mice (Figures 2B, C). Lastly, whereas we have previously reported that an increased frequency of TAM II+ and $\mathrm{CDC}$ accumulate in mammary tumors growing in Camkk2/- mice compared to WT (26), we didn't find statistically significant differences in the frequency of TAM II+/cDC in lymphoma microenvironment (data not shown).

To evaluate whether the failure to expand MDSCs has an important causative role in the mechanism of tumor suppression, Gr1+ MDSC, which include both subsets of MMDSC and G-MDSC cells were isolated from the spleens of E.G7-OVA-bearing WT and Camkk2-/- then adoptively transferred into Camkk2/- mice via tail vein injection. $\mathrm{Camkk2}^{-/-}$mice receiving sham tail vein injection were also included as a control group. EG7-OVA cells were then inoculated in the flank of CTR and MDSC-treated mice. EG-7OVA cells failed to grow robustly in the Camkk2/- control group (Figure 2D), while tumors developed at an equal rate in 
A

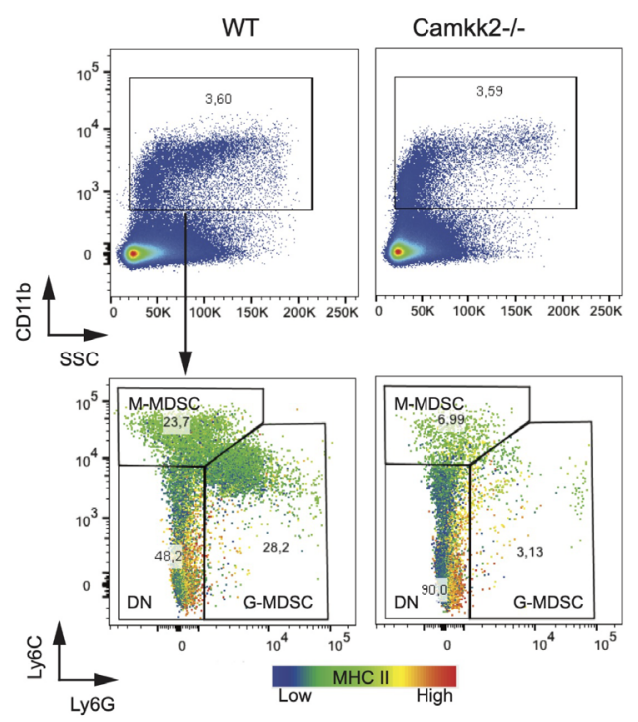

C

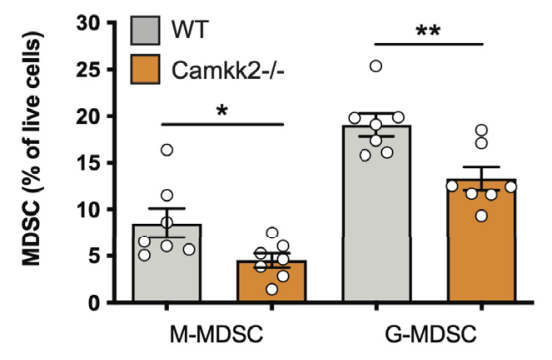

B
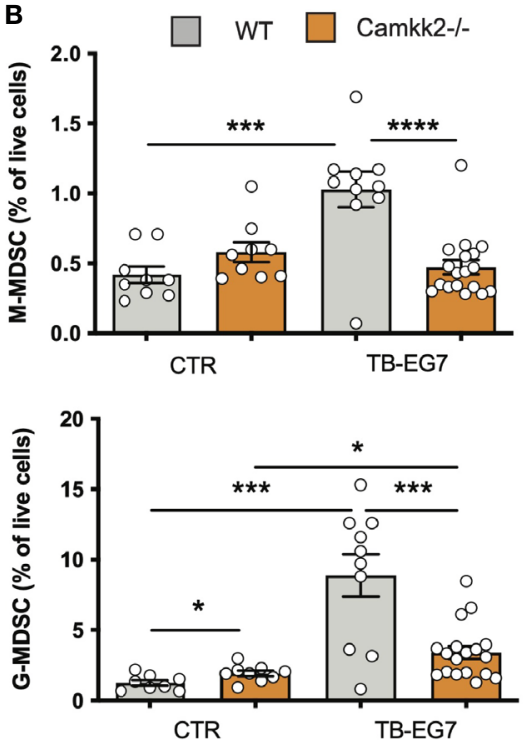

D

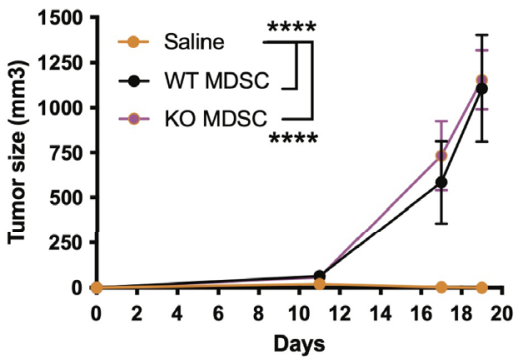

FIGURE 2 | Deletion of Camkk2 in the host prevents tumor-induced MDSC expansion. WT and Camkk2 ${ }^{-/-}$mice were injected with E.G7-OVA s.C. in the flank $\left(1 \times 10^{5}\right.$ cells/mouse). Spleens and tumors were removed, and single cell suspensions were stained for myeloid markers and analyzed by FACS. Groups of WT and Camkk2 $2^{-/}$mice not injected with tumor cells were used as control. (A) Gating strategy and representative plots of splenocytes isolated from tumor-bearing WT and Camkk2 $2^{-/-}$mice 17-21 days after injection. The heatmap indicates MHC II intensity in B and Ly6C intensity in C, respectively. (B) Percentages of M-MDSC, G-MDSC subsets in the spleen of normal and tumor-bearing mice (CTR and TB-EG7, respectively). (C) MDSCs in tumors removed from WT and Camkk2 ${ }^{-/-}$mice. (D) $1 \times 10^{4}$ E.G7-OVA were injected s.c. in the flank of Camkk $2^{-/-}$mice. At the same time, mice were injected IV with Gr1+ MDSC isolated from the spleen of tumor-bearing WT or Camkk2 ${ }^{-/-}$mice. The vehicle group received an equal volume of saline only. $\mathrm{N}=5$ mice/group. Repeated twice. ${ }^{*},{ }^{* \star}$, ${ }^{\star \star \star}$ and ${ }^{* \star \star \star}$ refer to $\mathrm{p}<0.05,0.01,0.005$, and 0.001 respectively.

$\mathrm{Camkk2}^{-/-}$mice that received either WT or Camkk2 $2^{-1-}$ MDSCs (Figure 2D). Although in our experimental conditions, the specific contribute of M- and G-MDSC subsets, these data indicate that restoring the number of MDSC was thus sufficient to revert the protective effect of Camkk2 deletion on tumor growth. These findings suggest that deletion of CaMKK2 might impair the ability of IMC to generate a sufficient number of MDSC required to block the immune response toward the lymphoma cells. On the other hand, the results from the adoptive transfer experiment also suggest that the deletion of CaMKK2 did not affect the capability of more mature MDSC to promote the tumor growth, suggesting that WT and Camkk2 $2^{-1-}$ MDSCs might have a comparable immunosuppressive activity, at the cellular level.

\section{Deletion of Camkk2 Impairs MDSC Expansion In Vitro}

To establish the cell-intrinsic function of CaMKK2 in MDSCs, we leveraged well-characterized models of in vitro bone marrow-derived MDSC (BM-MDSC) (40, 41). We first determined whether bone marrow cells isolated from WT and Camkk2 $2^{-/-}$mice have a comparable ability to generate MDSC. To this end, bone marrow cells were collected from WT and Camkk $2^{-/}$femurs and cultured with GM-CSF/IL-6 in the presence or absence of E.G7-OVA-conditioned medium (TCM). After 4 days, non-adherent cells were harvested and analyzed for phenotype and function. The expression of CaMKK2 at the mRNA and protein levels was assessed using 
qPCR and immunoblot, respectively (Supplementary Figures S3A, B), while phenotype and yield of MDSC subsets were evaluated by flow cytometry (Figures 3A, B). The results of these experiments indicated that CaMKK2 was expressed at detectable RNA and protein levels by in vitro generated MDSC and were not detected in Camkk2 $2^{-/-}$MDSC (Supplementary Figures S3A, B). Less MDSC were harvested from bone marrow Camkk2 $2^{-/-}$cultures compared to WT (Figures $3 \mathbf{A}$ C). Of note, this phenomenon was associated with an increased percentage of cells showing a phenotype of more differentiated myeloid cells $\left(\mathrm{CD} 11 \mathrm{~b}+, \mathrm{Ly} 6 \mathrm{C}^{\text {neg }}, \mathrm{Ly}_{6} \mathrm{G}^{\text {neg }}\right)$, which included more differentiated populations of non-adherent myeloid cells (e.g., dendritic cells; Figure 3B). To determine whether the effects of Camkk2 were reproducible in other experimental
A

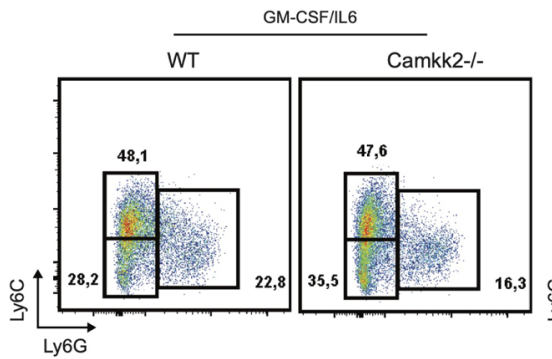

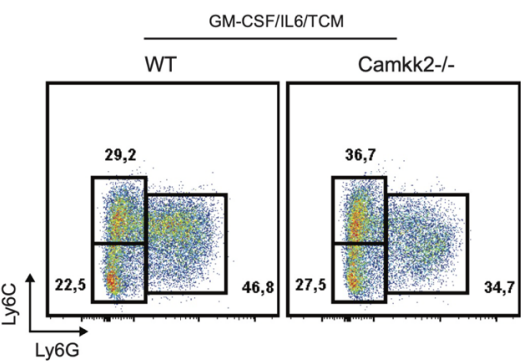

B

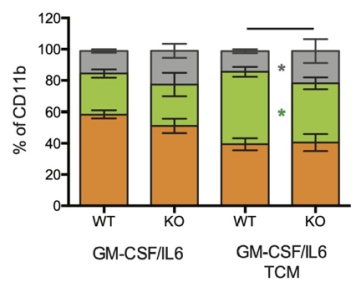

D

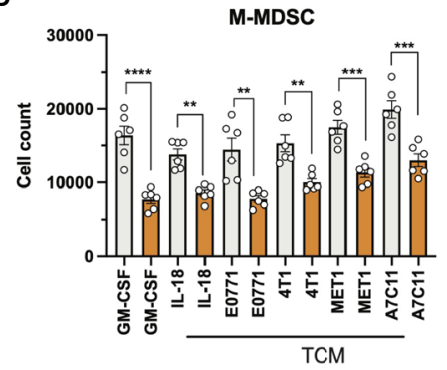

C

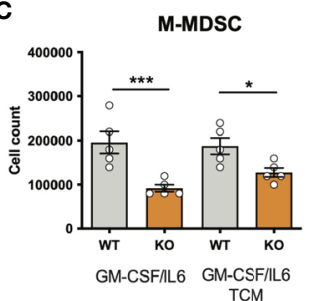

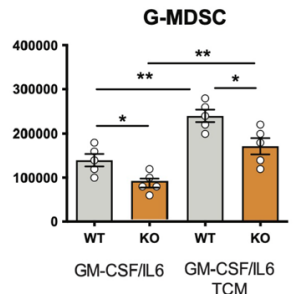
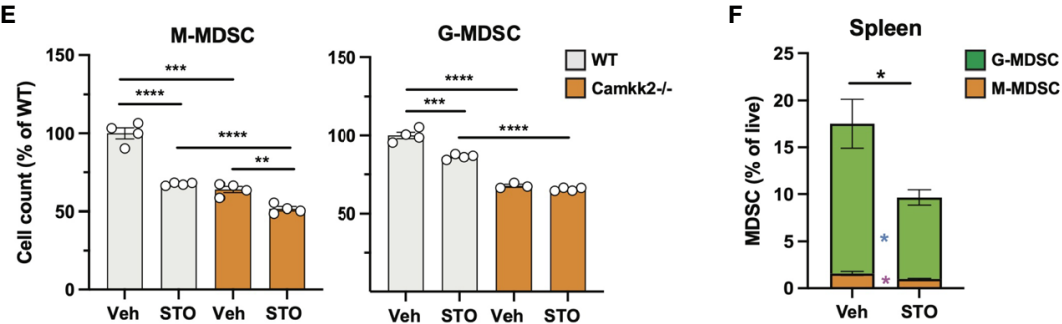

FIGURE 3 | Deletion of Camkk2 impairs MDSC expansion in vitro. Bone marrow nucleated cells from WT and Camkk $2^{-/}$mice were cultured with cytokines in the absence or presence of E.G7-OVA tumor conditioned medium (30\% v/v). After 4 days, cells were collected, stained, and analyzed by flow cytometry. (A) Gating strategy to identify MDSCs and representative dot plots. (B, C) Percentage and absolute number of MDSCs. (D). Yield of MDSC generated with GM-CSF in the presence of the cytokines and mammary tumor cells-TCM (E) Yield of MDSC generated in the presence of STO-609 (2uM). (F) EG7-OVA cells were inoculated SC in the flank of WT and Camkk2 ${ }^{-1-}$ mice. When the tumor where established, mice bearing tumors of comparable size (400-600 $\mathrm{mm}^{3}$ ) were treated with STO-609 (40 $\mu$ moles $/ \mathrm{Kg}$, IP at 48 hours interval, total 3-doses) and euthanized. Spleen were then removed and the frequency of MDSC were determined by flow cytometry. ${ }^{*},{ }^{\star *}$, ${ }^{* \star \star}$ and ${ }^{* \star \star *}$ refer to $\mathrm{p}<0.05,0.01,0.005$, and 0.001 , respectively. 
settings, BM cells from WT and Camkk2 $2^{-/-}$mice were cultured with GM-CSF in the presence or absence of IL-18, which has been identified as an important driver of MDSC-mediated immunosuppression $(42,43)$. In addition, MDSC from both genotypes were also generated in the presence or absence of TCM derived from several breast cancer cell lines, including EO771, 4T1, MET1, and A7C11. Consistent with the results from GM-CSF/IL-6 and EG7-OVA-conditioned medium, in all these experimental conditions, fewer MDSCs were generated from Camkk2 $2^{--}$BM cells compared to WT (Figure 3D). WT and Camkk2 ${ }^{-/-}$BM cells were then cultured with GM-CSF in the presence or absence of STO-609 $(2 \mu \mathrm{M})$ or vehicle. After 4 days cells were harvested, and the phenotype was determined by flow cytometry. With results from BM cells isolated from WT and Camkk2 $2^{-/-}$mice, the presence of STO-609 in the differentiation medium impaired the ability of WT BM cells to generate MDSCs (Figure 3E). In contrast, STO-609 slightly affected the yield of M-MDSC and exerted no significant changes on G-MDSC generated from Camkk2 ${ }^{-/-}$BM cells (Figure 3E). Lastly, we determined the effects of STO-609 on MDSC accumulating in the spleen of tumor-bearing mice. To this purpose, we inoculated EG7-OVA cells SC in the flank of WT and $\mathrm{Camkk2}^{-/-}$mice, as described in Figure 1E. When the tumor where established, mice bearing tumors of comparable size (400-600 $\left.\mathrm{mm}^{3}\right)$ were treated with STO-609 (40 $\mu$ moles $/ \mathrm{Kg}$, IP at 48 hours interval, total 3-doses) and euthanized. Spleen were then removed and the frequency of MDSC were determined by flow cytometry. As shown in the Figure 3F, the frequency of both M-MDSC and G-MDSC were significantly reduced in mice treated with STO-609 compared with vehicle. Overall, these findings indicate that Camkk2 inhibitors can be used to inhibit MDSC accumulation in vitro as well as in vivo.

The impact of Camkk2-deficiency on the immunosuppressive functions of MDSC was evaluated next. For these studies, MDSC were generated from BM of WT and Camkk2 ${ }^{-/-}$MDSC. After 4 days, MDSC were collected and co-cultured with CFSE-labeled T cells with or without anti-CD3/CD28 beads. Regardless of genotype, MDSC exerted significant suppressive effects on $\mathrm{T}$ cells, inhibiting proliferation, CD25, and CD69 upregulation induced by CD3/CD28 stimulation (Supplementary Figures S3C, D). Cumulatively, these data confirmed the in vivo tumor suppression results, indicating that the deletion of Camkk2 impacts MDSC expansion, exerting only a negligible effect on MDSC immunosuppressive function.

\section{Deletion of Camkk2 Impairs Survival and Enhances Terminal Differentiation of MDSC}

Multiple mechanisms could account for the decreased expansion of Camkk2 $2^{-/}$MDSC such as increased cell death or enhanced differentiation into mature myeloid cells (e.g. macrophages and dendritic cells). We first investigated whether the deletion of Camkk2 $2^{-/-}$increased the susceptibility of MDSC to apoptosis. Bone marrow cells from WT and Camkk2 $2^{-/-}$were cultured in the presence of GM-CSF and EG7-OVA-CM. After 4 days, MDSC were harvested, and apoptotic cells were identified by flow cytometry. As expected, less M-MDSC and G-MDSC were recovered from Camkk2/- $\mathrm{BM}$ cultures compared to WT (Figures 4A, B). Consistent with this result, higher percentages of early and late apoptotic cells (Annexin $V^{+} 7 \mathrm{ADD}^{-}$and Annexin $\mathrm{V}^{+} 7-\mathrm{AAD}^{+}$, respectively) were also detected in $\mathrm{Camkk2}^{-/-}$MDSC subsets compared to WT (Figure 4C). This finding prompted us to evaluate the capability of Camkk2 $2^{-1-}$ MDSC to survive during oxidative stress, a well-known inducer of cell death (44). MDSC cells were then exposed to hydrogen peroxide $\left(\mathrm{H}_{2} \mathrm{O}_{2}\right)$-induced oxidative injury and the percentage of apoptotic cells was monitored over time by flow cytometry. A progressive increase in either M-MDSC and G-MDSC apoptotic cells was observed following acute exposure to $\mathrm{H}_{2} \mathrm{O}_{2}$. Of note, the sensitivity of MDSC generated from Camkk2 ${ }^{-/-} \mathrm{BM}$ cells to the hydrogen peroxide oxidative stress- was significantly higher compared to WT (Figure 4D).

Data combined from multiple experiments indicated that, alongside a decreased yield of MDSC, more mature myeloid cells showing the typical phenotype of DC ( $\mathrm{Ly}^{-}, \mathrm{Ly}_{6 \mathrm{G}}^{-}$, $\mathrm{CD}_{11 \mathrm{c}^{+}}$, MHC $\mathrm{II}^{\text {high }}$ ) were generated from Camkk2 ${ }^{-/-} \mathrm{BM}$ cells cultures compared to WT (Figures 5A, B). Further, adherent cells recovered from WT BM cultures exhibited a typical M2-like morphology with significant elongation and branching while the cells recovered from Camkk2 $2^{--}$cultures displayed a round shape M1-like morphology (Supplementary Figure S4A). FACS and qPCR analyses corroborated this data, showing higher MHC II expression and lower ArgI and Chi3l3 mRNAs levels in the adherent cells recovered from Camkk2 $2^{-1-}$ cultures compared to WT cells (Supplementary Figures S4B, C). These findings indicated that, in addition to its effects on apoptosis, CaMKK2 functions to fine-tuning the terminal differentiation process of more mature MDSC. To directly evaluated this hypothesis, in vitro generated M-MDSC (Ly6C $^{\text {high }} \mathrm{Ly}_{6 \mathrm{G}}^{\mathrm{dim}} \mathrm{CD} 11 \mathrm{c}^{\text {neg }} \mathrm{MHC} \mathrm{II}^{\text {neg }}$ ) and G-MDSC (Ly6C $^{\text {dim }}$ Ly6G $^{\text {high }}$ CD11 $c^{\text {neg }}$ MHC II ${ }^{\text {neg }}$ ) were sorted by FACS and cultured with G-MCSF/IL6 in the presence of E.G7-OVA-TCM for additional 48 hours. Non-adherent cells were then harvested and analyzed by flow cytometry. As expected, the terminal differentiation of M-MDSC was associated with a consistent downregulation of Ly6C expression, leading to a remarkable accumulation of $\mathrm{LyC}^{\mathrm{dim} /}$ Ly6G ${ }^{\text {dim }}$ DN cells (Figures 5 C top panels, orange dots, 5D). Interestingly, in $\mathrm{DN}$ gate, more cells expressing the typical phenotype of dendritic cells (CD11 $\mathrm{c}^{+}$MHC II ${ }^{\text {high }}$ ) were generated from $\mathrm{Camkk2}^{-/-}$purified M-MDSC compared to WT (Figures 5C lower panels, orange dots, and $5 \mathbf{E}$ ). Regardless of genotype, the majority of cells recovered from G-MDSC cultures still expressed detectable levels of Ly6G and only a negligible number of them acquired DN/DC phenotypes (Figure 5C, green dots). Collectively, these findings indicated that deletion of Camkk2 accelerates the terminal differentiation of M-MDSC cells toward more mature myeloid cell subsets (e.g., M1 macrophages and dendritic cells) and this mechanism, in conjunction with increased apoptosis would account for the insufficient expansion of Camkk2 $2^{-/-}$MDSC. 
A

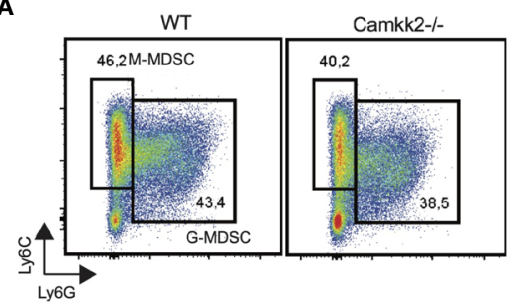

C
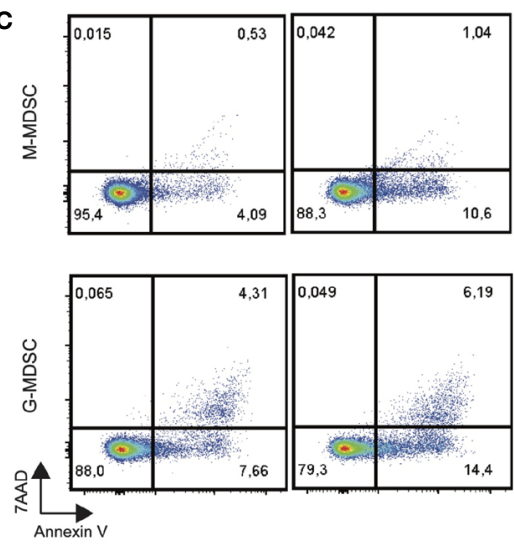

D

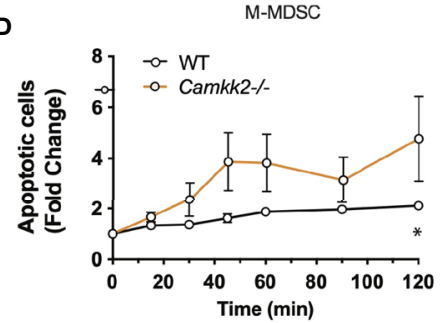

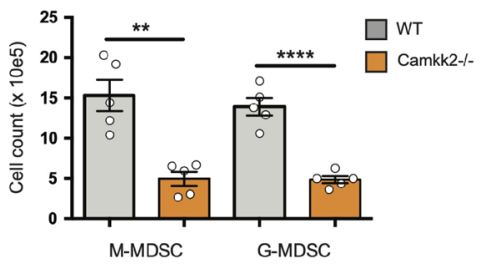
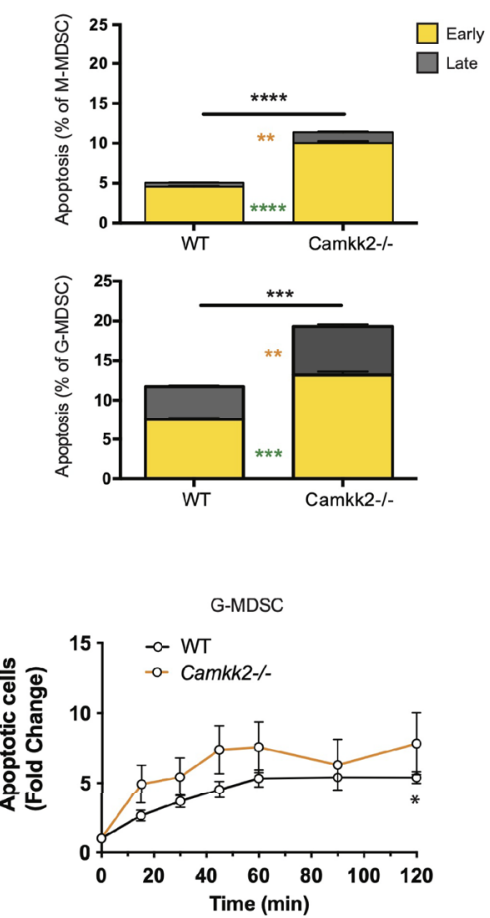

FIGURE 4 | Deletion of Camkk2 impairs the survival of MDSC. Bone marrow cells from WT and Camkk2 ${ }^{-1}$ mice were cultured with GM-CSF/IL6 cytokines in the absence or presence of E.G7-OVA tumor conditioned medium (30\% v/v). After 4 days, cells were collected, stained, and analyzed by flow cytometry. (A) Gating strategy and representative FACS profiles. (B) Yield of WT and Camkk2 ${ }^{-1-}$ M-MDSC, G-MDSC, and DC. (C) Percentage of early and late apoptotic MDSC. N $=5$ biological replicates/group. (D) Bone marrow nucleated cells from WT and Camkk2 $2^{-1-}$ mice were cultured with GM-CSF/L6 cytokines in the absence or presence of E.G7-OVA tumor conditioned medium ( $30 \% \mathrm{v} / \mathrm{v})$. MDSC were collected after 48 hours of culture and exposed to $1 \mu \mathrm{M} \mathrm{H}_{2} \mathrm{O}_{2}$. Apoptotic cells were identified by flow cytometry at the indicated time points. ${ }^{*},{ }^{* \star},{ }^{* * *}$, and ${ }^{* \star \star *}$ refer to $p<0.05, p<0.01, p<0.001, p<0.0001$, respectively. T-test and two-ways ANOVA were used to calculate $p$-values in panels $B$ and $D$, respectively.

\section{Camkk2 Deficiency Increases Reactive Oxygen Species (ROS) Accumulation and Impairs Mitochondria Functions in MDSC}

ROS are important regulators of multiple cellular processes including cell death, proliferation, and differentiation (44, 45). Physiological levels of ROS are required for MDSC generation (46), while genetic alterations causing abnormal accumulation of ROS trigger MDSC apoptosis, which prevents tumor-induced MDSC expansion $(47,48)$. Increasing evidence documents a network of relevant bidirectional interactions among ROS and calcium signaling (49-51), and CaMKK2 has been recently identified as an important regulator of ROS accumulation and ferroptosis in tumor cells (52). These findings prompted us to determine whether deletion of Camkk2 interferes with the mechanism of ROS accumulation in MDSCs. To test this, MDSCs were generated from BM cells of WT and Camkk2 ${ }^{-1}$ mice, and ROS accumulation was evaluated by flow cytometry (Supplementary Figure S5A). The results of these experiments indicated that higher ROS levels accumulated in Camkk2-1MDSC compared to WT (Figure 6A). We next evaluated whether deletion of Camkk2 interferes with the expression of genes involved in ROS production (NADPH Oxidase 1 and 2; Nox1 and Nox2, respectively) or in the antioxidant response (Catalase and nuclear factor erythroid 2-related factor-2, Cat and Nrf2, respectively). Genes positively regulating ROS accumulation (Nox1 and Nox2) were expressed at comparable (Nox1) or lower levels (Nox2) in Camkk2 $2^{-/-}$MDSC compared to WT (Supplementary Figure S5B). Moreover, lower levels of Cat 


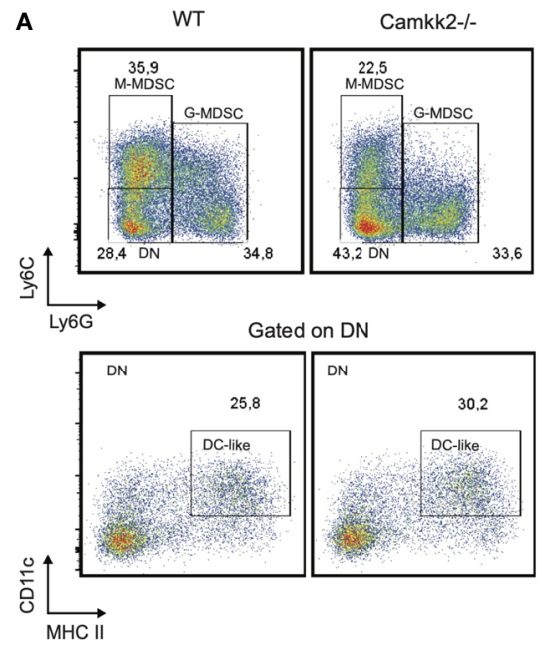

B
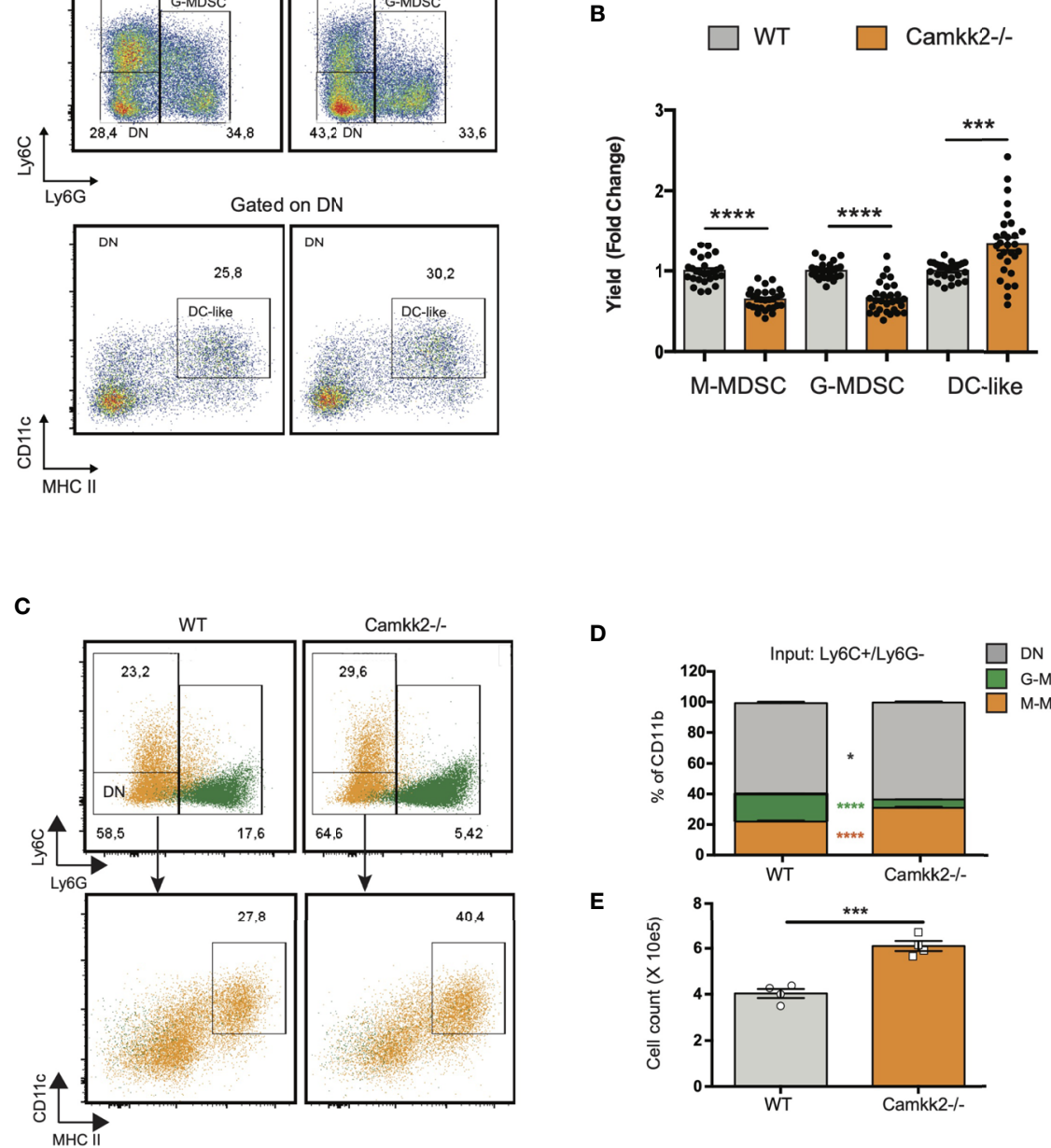

D

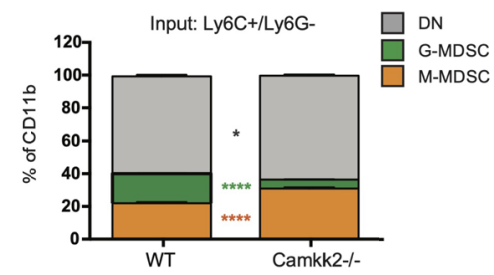

E

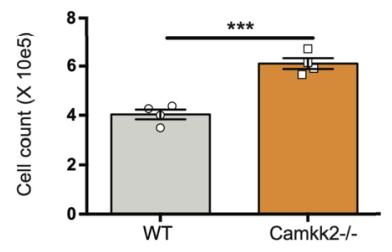

FIGURE 5 | Deletion of Camkk2 accelerates the terminal differentiation of MDSCs. Bone marrow cells from WT and Camkk2 ${ }^{-/}$mice were cultured with GM-CSF/L6 cytokines in the absence or presence of E.G7-OVA tumor conditioned medium (30\% v/v). After 4 days, cells were collected, stained, and analyzed by flow cytometry. (A) Gating strategy and representative FACS profiles. (B) Yield of M-MDSC, G-MDSC and DC. Bar graph showing the cumulative results from 6 independent experiments and fold changes $(\mathrm{FC})$ have been calculated on the yield of WT MDSC. (C, D) Bone marrow cells from WT and Camkk $2^{-1-}$ mice were cultured with GM-CSF/IL6 cytokines in the absence or presence of E.G7-OVA tumor conditioned medium (30\% v/v). After 4 days, non-adherent cells were collected and sorted by flow cytometry. Purified M-MDSC and G-MDSC were then cultured in the presence of GM-CSF/L-6 and $30 \%$ of EG7 CM for additional 48 hours and analyzed by flow cytometry. (C) Gating strategy to identify M-MDSC, G-MDSC, and DC in M-MDSC and G-MDSC cultures. Overlapping dot plots refer to the FACS profiles of cells collected from M-MDSC and G-MDSC (shown in orange and green, respectively). (D) Percentage of M-MDSC, G-MDSC, and DN subsets (upper).

(E) Yield of Ly6C- Ly6G- CD11 $\mathrm{C}^{+} \mathrm{MHC} \mathrm{II}^{+}$cells (namely DC). ${ }^{*}$, ${ }^{* \star \star}$ and ${ }^{\star \star \star \star}$ refer to $\mathrm{p}<0.05,0.005$ and $\mathrm{p}<0.001$, respectively.

and Nfr2 mRNAs were expressed in Camkk2 ${ }^{-/-}$MDSC compared to WT (Figure 6B and Supplementary Figure S5B). These findings, alongside the higher susceptibility of Camkk2 $2^{-/-}$ MDSC to oxidative stress-induced apoptosis (Figure 4D), indicated that CaMKK2 could function by regulating the antioxidant response in MDSC. To evaluate the functional role of ROS, MDSC were generated from BM of WT and Camkk2 $2^{-/}$ mice in the presence or absence of hydrogen peroxide (44) or butylated hydroxyanisole (BHA), which is a known scavenger of ROS (53). The presence of hydrogen peroxide in the culture medium impaired the production of WT MDSC, with no significant effects on Camkk2 $2^{-/-}$M-MDSC (Supplementary Figure S5C upper). Further, regardless of the genotype, the yield of G-MDSC and DC was negatively regulated by prolonged exposure to $\mathrm{H}_{2} \mathrm{O}_{2}$ (Supplementary Figure S5C upper). On the contrary, more MDSC and less DC were recovered from the culture of WT bone marrow cells treated with BHA (Supplementary Figure S5C, lower). However, only minor effects were induced by this ROS scavenger on Camkk $2^{-/-}$ MDSC and DC (Supplementary Figure S5C, lower). We lastly 

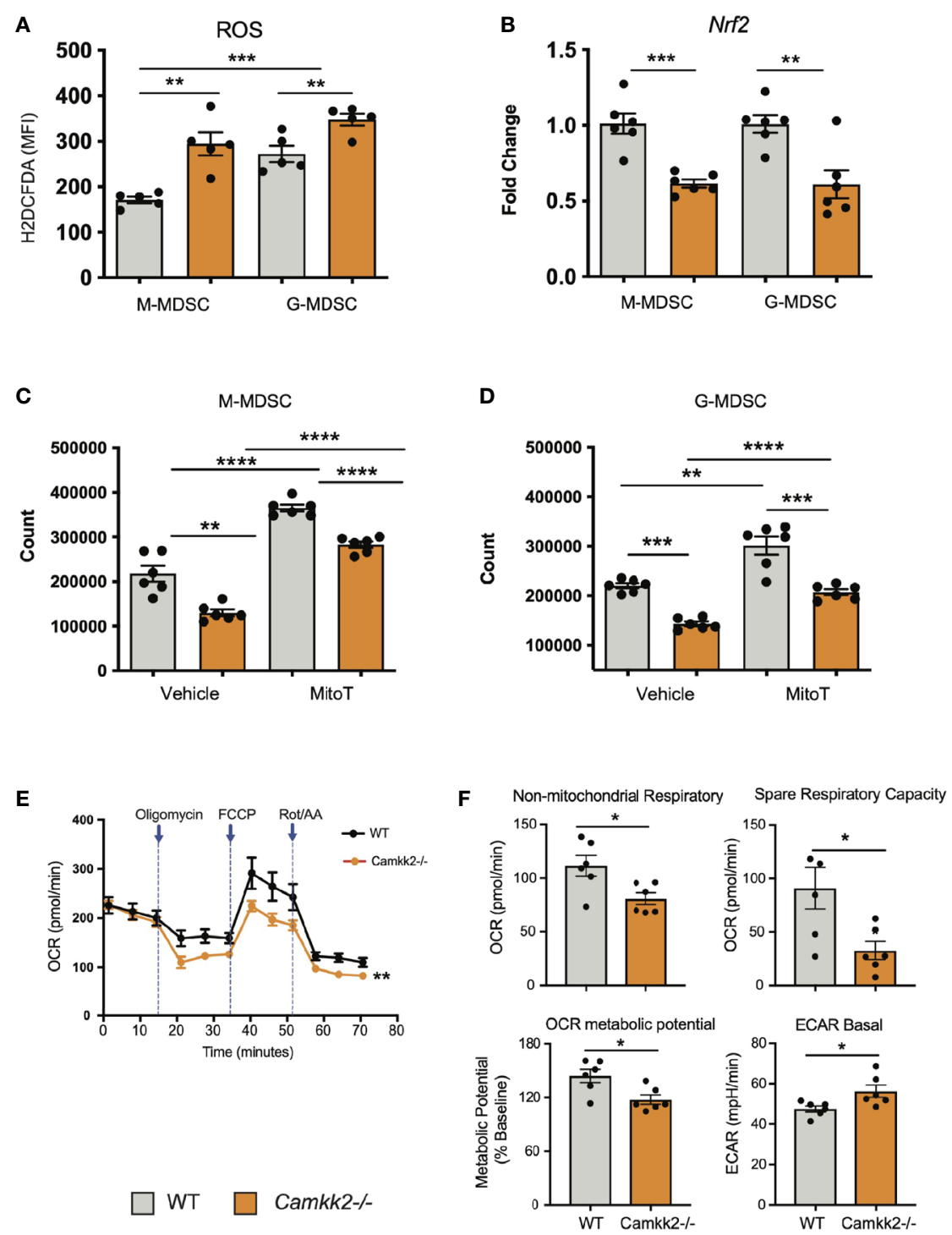

FIGURE 6 | Camkk2 deficiency increases reactive oxygen species (ROS) accumulation and impairs mitochondria functions in MDSC. MDSCs were generated from bone marrow nucleated cells of WT and Camkk2 $2^{-1-}$ mice femurs. (A) MDSCs were identified according to the gating strategy described previously and ROS levels were evaluated by flow cytometry using H2DCFDA staining. (B) M-MDSC and G-MDSC were purified by sorting and Nrf2 expression was determined by qPCR. (C, D) Yield of M-MDSC and G-MDSC generated in the presence of 20 $\mu$ M Mito Tempo, a mitochondrial ROS scavenger compound, added on Day 0 and Day 3. (E, F) Metabolic analysis of in vitro generated MDSC using Seahorse XF24 Analyzer. (E) OCR response to oligomycin, FCCP, and Rot/AA. (F) Quantification of nonmitochondrial respiratory, spare respiratory capacity, OCR metabolic potential, and ECAR basal level calculated by Agilent Seahorse Wave Software. Combined from two experiments. $\mathrm{N}=6$. The experiment was repeated twice. ${ }^{*}{ }^{\star \star \star}{ }^{* \star *}$, and ${ }^{\star \star \star \star *}$ refer to $\mathrm{p}<0.05, \mathrm{p}<0.01, \mathrm{p}<0.001, \mathrm{p}<0.0001$, respectively.

tested the ability of MitoTempo, a specific scavenger of mitochondrial superoxide (54) to interfere with the generation of MDSC. As predicted by our hypothesis, more MDSC were recovered from WT MDSC treated with this ROS scavenger (Figures 6C, D). Interestingly, the targeting of the mitochondrial source of ROS with MitoTempo also increased the yield of $\mathrm{Camkk2}^{-/-}$M-MDSC and G-MDSC cells (Figures 6C, D). On the other hand, treatment with MitoTempo was associated with a decreased yield of WT and Camkk2 $2^{-/-}$DC (Supplementary Figure S5D).

The relevant role of mitochondria as a source and target of ROS $(55,56)$ in conjunction with the results from MitoTempo experiments (Figures 6C, D), suggests the hypothesis that deletion of Camkk2 may have a relevant impact on metabolic mitochondria functions. To investigate this hypothesis, MDSC were generated from WT and Camkk2 $2^{-/-}$BM cells, and Agilent 
Seahorse XF technology was leveraged to analyze mitochondria metabolic functions. WT and Camkk2 ${ }^{-/-}$MDSC cells showed a comparable basal oxygen consumption rate (OCR), while the OCR of Camkk2 $2^{-/-}$MDSC was significantly lower compared to WT MDSC, after oligomycin and FCCP challenge (Figures 6E, F). The lower non-mitochondrial respiratory rate associated with a decreased spare respiratory capacity and OCR metabolic potential were found in Camkk2 $2^{--}$MDSC compared to WT (Figures 6E, F). Lastly, genetic deletion of Camkk2 was also associated with increased ECAR basal level (Figures 6E, F). In aggregate, these findings indicated WT and Camkk2 ${ }^{-/-}$MDSCs had distinct metabolic profiles, with a higher reliance of Camkk2 $2^{-/}$MDSCs on mitochondrial respiration for energy generation, compared to WT MDSCs.

\section{AMPK Pathway Is the Downstream Target of CaMKK2 in Regulating Cellular ROS Accumulation}

AMPK is a master regulator of ROS metabolism and mitochondrial functions, and the functional crosstalk between AMPK signaling and mitochondria has a key role in the control of cellular metabolism $(18,19,57,58)$. Indeed, mitochondriaderived ROS can activate AMPK signaling, which in turn can stimulate the antioxidant response to fine-tuning mitochondrial ROS production $(57,59)$. Deletion of CaMKK2 may impair this regulatory circuit, leading to a dysregulated response to oxidative stress, ROS accumulation, and mitochondria dysfunction. To verify this hypothesis, we measured the levels of total AMPK and phospho-Thr172-AMPK (pAMPK) in MDSCs generated from WT and Camkk2 $2^{-/-}$BM cells. Comparable levels of AMPK were detected in WT and Camkk2 ${ }^{-/-}$MDSCs, while significantly lower levels of phospho-Thr172-AMPK (pAMPK) accumulated in Camkk2 $2^{-/-}$MDSCs (Figure 7A). To further evaluate the role of CaMKK2 in MDSC signaling, BM-MDSC generated from WT and Camkk2 ${ }^{-/-}$BM cells were collected at day 3 and starved for additional 12 hours from cytokines and E.G7-OVA TCM. These cells were then stimulated with IL-6 to trigger the accumulation of phospho-STAT3 (pSTAT3), which is an important regulator of MDSC development $(60,61)$. Regardless of genotype, pSTAT3 accumulated at comparable levels following IL-6 stimulation (Figure 7B). In addition, pAMPK levels were not modulated by IL-6 and were remarkably higher in WT MDSCs than $\mathrm{Camkk}^{-/-}$MDSCs. To determine whether the failure to stimulate the AMPK signaling may account for ROS accumulation in Camkk2 $2^{-/-}$MDSCs, intracellular ROS were evaluated by flow cytometry in MDSC treated with the AMPK agonist compound AICAR (62). Under basal conditions, higher levels of ROS accumulated in untreated Camkk2 $2^{-/-}$MDSCs compared to WT (Figures 7C, D). Treatment with the AMPK agonist did not induce any significant changes in WT MDSC, while significantly lower levels of ROS were detected in Camkk2 $2^{--}$MDSC treated with AICAR compared to Camkk2 $2^{-/}$ MDSC exposed to the vehicle alone (Figures 7C, D). These results indicated that restoring AMPK signaling is sufficient to compensate for the effects of genetic deletion of Camkk2 on ROS, pinpointing the CaMKK2-AMPK axis as an important component of the mechanism regulating the antioxidant response in MDSC.

\section{DISCUSSION}

Herein, we demonstrate CaMKK2 expression in host cells regulates syngeneic tumor growth by controlling tumorinduced MDSCs. We show syngeneic lymphoma cells fail to survive in mice genetically deleted of Camkk2 and this is reversed by adoptive transfer of MDSCs from spleens of tumor-bearing mice. The ability to suppress $\mathrm{T}$ cell responses is a hallmark of MDSCs, and this remarkable function largely accounts for their inhibitory effects on anti-tumor immune response (5). We found that CaMKK2 is highly expressed in M-MDSC and is also detectable in G-MDSC. As previously reported in breast tumor models (26), deletion of Camkk2 in the host is also associated with enhanced $\mathrm{T}$ cell response toward an antigen expressed in lymphoma cells. Here, we demonstrated that the failure of lymphoma cells to grow in Camkk2 $2^{-/-}$mice is associated with a decreased accumulation of MDSCs, while the adoptive transfer of MDSC is sufficient to restore the ability of tumor cells to grow in CaMKK2 deficient mice. These findings identify CaMKK2 as an important component of the mechanism regulating the expansion of MDSC in tumor-bearing individuals.

The expansion of immature myeloid cells (IMC) is induced by a variety of tumor-released soluble factors that, in combination with cytokines active on common myeloid progenitors, re-shape the normal myeloid differentiation process (5). According to the classical 2-signals model, STAT-3 activating cytokines leads to increased survival and proliferation of IMC through upregulation of B-cell lymphoma XL, (BCL-XL), cyclin D1, $\mathrm{MYC}$, and survivin. Inflammatory molecules then provide an activation signal 2, which supports the expansion and differentiation of MDSCs (63). Finally, additional signals drive the terminal differentiation of M-MDSCs in tumor-associated macrophages (64). Our data indicate that CaMKK2 controls critical steps of this process. First, we demonstrated that bone marrow cells from Camkk2 $2^{-/-}$mice have a decreased ability to generate MDSCs and this phenomenon is largely dependent on an increased susceptibility of Camkk2 $2^{-/-}$MDSCs to apoptosis. Second, we show that purified M-MDSC generated from Camkk2/- bone marrow cells have an increased ability to differentiate toward DC lineage. In aggregate, these data indicate that CaMKK2 functions as an important checkpoint to control the survival of IMC and the terminal differentiation of MDSC.

In the hematopoietic compartment, ROS work as a rheostat to regulate the differentiation of hematopoietic stem and progenitor cells (47). While low levels of ROS are critical to promoting differentiation of common myeloid progenitors (CMP) toward MEP, high levels of ROS can trigger apoptosis in more sensitive hematopoietic cell types or drive CMP to differentiate toward GMP or eventually IMC, in tumor-bearing individuals. We demonstrated that Camkk2 $2^{-/-}$MDSC accumulate more ROS, are more susceptible to oxidative stress-induced apoptosis, and 
A
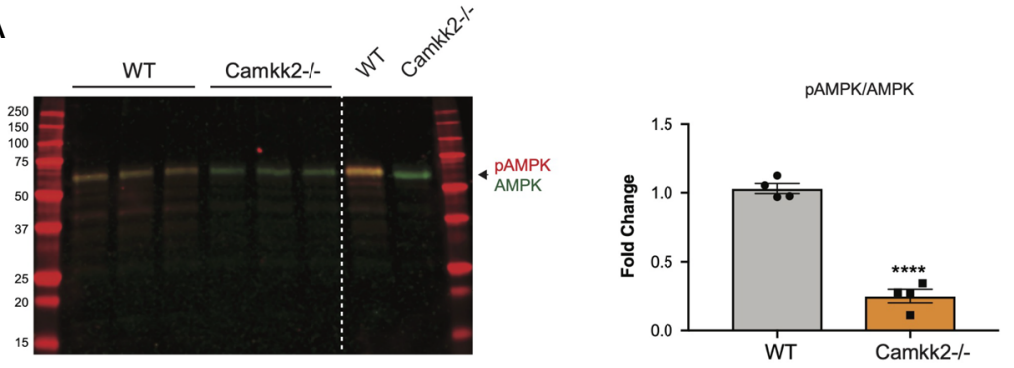

B
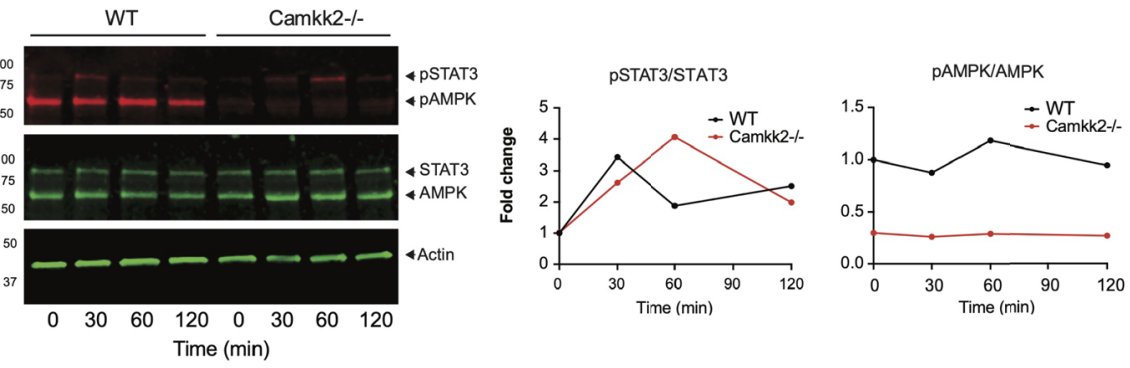

C

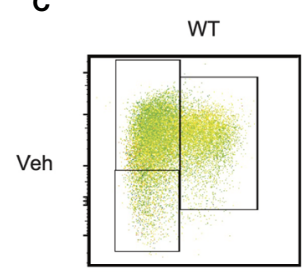

Camkk2-1-
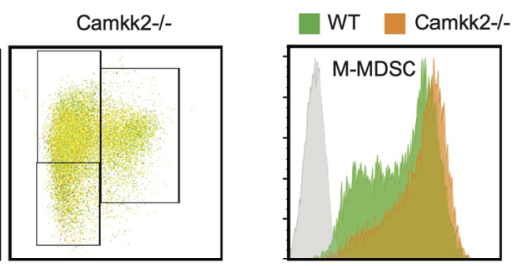

D
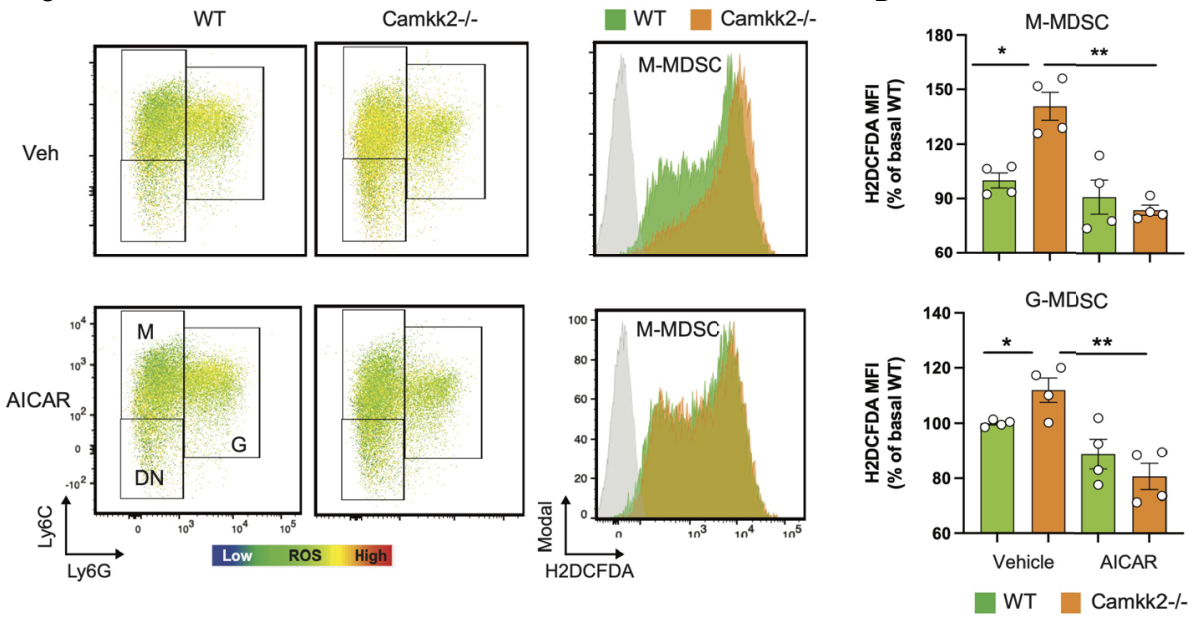

FIGURE 7 AMPK pathway is the downstream target of CaMKK2 in regulating cellular ROS accumulation. MDSCs were generated in vitro from WT and CaMKK2 ${ }^{-1}$ BM. (A) Immunoblots of WT and Camkk $2^{-/-}$and quantitation of phospho-AMPK (pAMPK) levels (left and right, respectively). (B) in vitro generated MDSC were collected at day 4, washed, and starved 12 hours from cytokines and tumor conditioned mix. MDSC were then recovered and cultured in the presence of IL-6 for the reported time. PSTAT3 and PAMPK immunoblots and quantitation (left and right, respectively). (C) in vitro generated MDSCs were treated with AICAR, (125 $\mu$ M) or an equal volume of vehicle, for 2 hours. MDSC were then collected and stained for surface markers. ROS were detected by H2DCFDA staining. [(C), left panels] representative heatmap dot plots and gating strategy to identify M-MDSC, G-MDSC, and DN subsets. [(C), right panels] Representative profiles of H2DFDA staining in WT and Camkk2-/- MDSC (green and orange profiles, respectively). H2DFDA unstained WT MDSC were used as negative control (Grey profiles). (D) Expression of H2DFDA mean fluorescence intensity (MFI) detected in WT and KO MDSC. Four biological replicates are reported. The results are expressed as percentage basal (average of H2DFDA MFI detected in vehicle-treated WT MDSC). *, ${ }^{* *}$ and ${ }^{* \star * *}$ refer to $\mathrm{p}<0.05 ; 0.01$ and 0.001 , respectively.

have a better ability to terminally differentiate into DC compared to WT. The deletion of Camkk2 is associated with alterations of mitochondrial functions, while the treatment with a pharmacological inhibitor of mitochondria-generated ROS partially counterbalances the loss of Camkk2 in MDSC. Our data also indicate that deletion of Camkk2 is associated with a downregulation of $\mathrm{Nrf2}$, which is a master regulator of the antioxidant response (65) and mitochondrial ROS production
(66). Accordingly, increased susceptibility to apoptosis with diminished production of MDSC has been reported in $\mathrm{Nrf2}^{-/-}$ mice (47). In aggregate, these findings, in conjunction with the ability of CaMKK2 to regulate the NRF2 signaling and in turn ferroptosis in cancer cells (52), pinpoint $N r f 2$ as a potential relevant downstream target of CaMKK2 in MDSC.

AMPK is a relevant proximal target of CaMKK2 and a master regulator of the metabolic and oxidative stress response. 
Consequently, the decreased ability of Camkk2 $2^{-/-}$MDSC to activate AMPK might account for the elevated ROS levels. The ability of AMPK agonist to revert the effects of Camkk2 deletion on ROS accumulation, support the hypothesis that AMPK signaling has an important role in this mechanism. Further, AMPK has also an important role in the mechanisms of mitochondria adaptation to stress, and the failure to activate AMPK signaling might also account for the decreased ability of $\mathrm{Camkk2}^{-/-}$MDSC to adapt mitochondria functions to metabolic stress. Although we cannot establish if mitochondria defects are primarily caused by the CaMKK2 deficiency or the consequence of high ROS levels, our data identify CaMKK2 as an important regulator of ROS metabolism and mitochondria bioenergetics in MDSC.

In summary, our data indicate that CaMKK2 has a cellautonomous function in MDSCs, identifying the CaMKK2AMPK axis as an important regulator of intracellular ROS levels and mitochondrial bioenergetics. The use of a small molecule, STO-609 recapitulates much of the genetic knock-out models. Translationally, we propose CaMKK2 as an important regulator of MDSCs biology, and a potential therapeutic target to stimulate the anti-tumor immune response.

\section{DATA AVAILABILITY STATEMENT}

The raw data supporting the conclusions of this article will be made available by the authors, without undue reservation.

\section{ETHICS STATEMENT}

The animal study was reviewed and approved by Duke University Institutional Animal Care and Use Committee.

\section{REFERENCES}

1. Gabrilovich DI, Bronte V, Chen SH, Colombo MP, Ochoa A, OstrandRosenberg S, et al. The Terminology Issue for Myeloid-Derived Suppressor Cells. Cancer Res (2007) 67(1):425. doi: 10.1158/0008-5472.CAN-06-3037

2. Veglia F, Sanseviero E, Gabrilovich DI. Myeloid-Derived Suppressor Cells in the Era of Increasing Myeloid Cell Diversity. Nat Rev Immunol (2021) 21:485-98. doi: 10.1038/s41577-020-00490-y

3. Alshetaiwi H, Pervolarakis N, McIntyre LL, Ma D, Nguyen Q, Rath JA, et al. Defining the Emergence of Myeloid-Derived Suppressor Cells in Breast Cancer Using Single-Cell Transcriptomics. Sci Immunol (2020) 5(44). doi: 10.1126/sciimmunol.aay6017

4. Bronte V, Brandau S, Chen SH, Colombo MP, Frey AB, Greten TF, et al. Recommendations for Myeloid-Derived Suppressor Cell Nomenclature and Characterization Standards. Nat Commun (2016) 7:12150. doi: 10.1038/ncomms12150

5. Tcyganov E, Mastio J, Chen E, Gabrilovich DI. Plasticity of Myeloid-Derived Suppressor Cells in Cancer. Curr Opin Immunol (2018) 51:76-82. doi: 10.1016/j.coi.2018.03.009

6. Corzo CA, Cotter MJ, Cheng P, Cheng F, Kusmartsev S, Sotomayor E, et al. Mechanism Regulating Reactive Oxygen Species in Tumor-Induced MyeloidDerived Suppressor Cells. J Immunol (2009) 182(9):5693-701. doi: 10.4049/ jimmunol.0900092

\section{AUTHOR CONTRIBUTIONS}

$\mathrm{WH}, \mathrm{YL}, \mathrm{WL}, \mathrm{ES}, \mathrm{YZ}$, and PS performed in vivo and in vitro experiments with tumors and MDSC. AL and JM designed and performed experiments to assess metabolic changes in MDSC. MB designed and performed Elispots experiments. WH, NC, and LR designed the study and analyzed results. WH and LR prepared the manuscript. YK, LR, and NC revised the manuscript. All authors contributed to the article and approved the submitted version.

\section{FUNDING}

This work was supported by grants CA140307, Department of Defense (NC and LR), 1R01CA218442-01 (LR), 2U19AI06779816 (NC and LR) and W81XWH-20-1-0498 (LR).

\section{ACKNOWLEDGMENTS}

We thank Dr. Yiping for providing the GFP-luciferin transfected E.G7-OVA cell line; Dr. Benny Chen and all members of Nelson Chao's lab for criticisms and helpful discussion of the experimental data; Ching-Yi Chang and Debarati Mukherjee for providing breast cancer cells; Duke Human Vaccine Institute Flow Facility and Duke Cancer Institute Flow Cytometry Shared Resources for cell sorting.

\section{SUPPLEMENTARY MATERIAL}

The Supplementary Material for this article can be found online at: https://www.frontiersin.org/articles/10.3389/fimmu.2021.754083/ full\#supplementary-material

7. Raber PL, Thevenot P, Sierra R, Wyczechowska D, Halle D, Ramirez ME, et al. Subpopulations of Myeloid-Derived Suppressor Cells Impair T Cell Responses Through Independent Nitric Oxide-Related Pathways. Int J Cancer (2014) 134(12):2853-64. doi: 10.1002/ijc.28622

8. Kusmartsev S, Nefedova Y, Yoder D, Gabrilovich DI. Antigen-Specific Inhibition of CD8+ T Cell Response by Immature Myeloid Cells in Cancer Is Mediated by Reactive Oxygen Species. J Immunol (2004) 172(2):989-99. doi: 10.4049/jimmunol.172.2.989

9. Srivastava MK, Sinha P, Clements VK, Rodriguez P, Ostrand-Rosenberg S. Myeloid-Derived Suppressor Cells Inhibit T-Cell Activation by Depleting Cystine and Cysteine. Cancer Res (2010) 70(1):68-77. doi: 10.1158/00085472.CAN-09-2587

10. Condamine T, Mastio J, Gabrilovich DI. Transcriptional Regulation of Myeloid-Derived Suppressor Cells. J Leukoc Biol (2015) 98(6):913-22. doi: 10.1189/jlb.4RI0515-204R

11. Sato Y, Shimizu K, Shinga J, Hidaka M, Kawano F, Kakimi K, et al. Characterization of the Myeloid-Derived Suppressor Cell Subset Regulated by NK Cells in Malignant Lymphoma. Oncoimmunology (2015) 4(3):e995541. doi: 10.1080/2162402X.2014.995541

12. Zhu J, Huang X, Yang Y. Myeloid-Derived Suppressor Cells Regulate Natural Killer Cell Response to Adenovirus-Mediated Gene Transfer. J Virol (2012) 86 (24):13689-96. doi: 10.1128/JVI.01595-12 
13. Marvel D, Gabrilovich DI. Myeloid-Derived Suppressor Cells in the Tumor Microenvironment: Expect the Unexpected. J Clin Invest (2015) 125(9):335664. doi: 10.1172/JCI80005

14. Gabrilovich DI, Nagaraj S. Myeloid-Derived Suppressor Cells as Regulators of the Immune System. Nat Rev Immunol (2009) 9(3):162-74. doi: 10.1038/ nri2506

15. Fleming V, Hu X, Weber R, Nagibin V, Groth C, Altevogt P, et al. Targeting Myeloid-Derived Suppressor Cells to Bypass Tumor-Induced Immunosuppression. Front Immunol (2018) 9:398. doi: 10.3389/ fimmu.2018.00398

16. Berridge MJ, Bootman MD, Roderick HL. Calcium Signalling: Dynamics, Homeostasis and Remodelling. Nat Rev Mol Cell Biol (2003) 4(7):517-29. doi: $10.1038 / \mathrm{nrm} 1155$

17. Takemoto-Kimura S, Suzuki K, Horigane SI, Kamijo S, Inoue M, Sakamoto M, et al. Calmodulin Kinases: Essential Regulators in Health and Disease. J Neurochem (2017) 141(6):808-18. doi: 10.1111/jnc.14020

18. Hawley SA, Pan DA, Mustard KJ, Ross L, Bain J, Edelman AM, et al. Calmodulin-Dependent Protein Kinase Kinase-Beta is an Alternative Upstream Kinase for AMP-Activated Protein Kinase. Cell Metab (2005) 2 (1):9-19. doi: 10.1016/j.cmet.2005.05.009

19. Woods A, Dickerson K, Heath R, Hong SP, Momcilovic M, Johnstone SR, et al. Ca2+/calmodulin-Dependent Protein Kinase Kinase-Beta Acts Upstream of AMP-Activated Protein Kinase in Mammalian Cells. Cell Metab (2005) 2(1):21-33. doi: 10.1016/j.cmet.2005.06.005

20. Hardie DG. AMPK-sensing Energy While Talking to Other Signaling Pathways. Cell Metab (2014) 20(6):939-52. doi: 10.1016/j.cmet.2014.09.013

21. Blagih J, Coulombe F, Vincent EE, Dupuy F, Galicia-Vázquez G, Yurchenko E, et al. The Energy Sensor AMPK Regulates T Cell Metabolic Adaptation and Effector Responses. vivo Immun (2015) 42(1):41-54. doi: 10.1016/ j.immuni.2014.12.030

22. Racioppi L, Means AR. Calcium/Calmodulin-Dependent Protein Kinase Kinase 2: Roles in Signaling and Pathophysiology. J Biol Chem (2012) 287 (38):31658-65. doi: 10.1074/jbc.R112.356485

23. Teng EC, Racioppi L. Means AR. A Cell-Intrinsic Role for CaMKK2 in Granulocyte Lineage Commitment and Differentiation. J Leukoc Biol (2011) 90(5):897-909. doi: 10.1189/jlb.0311152

24. Racioppi L, Lento W, Huang W, Arvai S, Doan PL, Harris JS, et al. Calcium/ calmodulin-Dependent Kinase Kinase 2 Regulates Hematopoietic Stem and Progenitor Cell Regeneration. Cell Death Dis (2017) 8. doi: 10.1038/ cddis.2017.474

25. Racioppi L, Noeldner PK, Lin F, Arvai S, Means AR. Calcium/calmodulinDependent Protein Kinase Kinase 2 Regulates Macrophage-Mediated Inflammatory Responses. J Biol Chem (2012) 287(14):11579-91. doi: $10.1074 /$ jbc.M111.336032

26. Racioppi L, Nelson ER, Huang W, Mukherjee D, Lawrence SA, Lento W, et al. CaMKK2 in Myeloid Cells is a Key Regulator of the Immune-Suppressive Microenvironment in Breast Cancer. Nat Commun (2019) 10(1):2450. doi: 10.1038/s41467-019-10424-5

27. Gong S, Zheng C, Doughty ML, Losos K, Didkovsky N, Schambra UB, et al. A Gene Expression Atlas of the Central Nervous System Based on Bacterial Artificial Chromosomes. Nature (2003) 425(6961):917-25. doi: 10.1038/ nature 02033

28. Anderson KA, Ribar TJ, Lin F, Noeldner PK, Green MF, Muehlbauer MJ, et al. Hypothalamic CaMKK2 Contributes to the Regulation of Energy Balance. Cell Metab (2008) 7(5):377-88. doi: 10.1016/j.cmet.2008.02.011

29. Anderson KA, Lin F, Ribar TJ, Stevens RD, Muehlbauer MJ, Newgard CB, et al. Deletion of CaMKK2 From the Liver Lowers Blood Glucose and Improves Whole-Body Glucose Tolerance in the Mouse. Mol Endocrinol (2012). doi: 10.1210/me.2011-1299[published Online First: 2012/01/14

30. Le Naour A, Koffi Y, Diab M, Le Guennec D, Rougé S, Aldekwer S, et al. EO771, the First Luminal B Mammary Cancer Cell Line From C57BL/6 Mice. Cancer Cell Int (2020) 20:328. doi: 10.1186/s12935-020-01418-1

31. Lelekakis M, Moseley JM, Martin TJ, Hards D, Williams E, Ho P, et al. A Novel Orthotopic Model of Breast Cancer Metastasis to Bone. Clin Exp Metastasis (1999) 17(2):163-70. doi: 10.1023/a:1006689719505

32. Borowsky AD, Namba R, Young LJ, Hunter KW, Hodgson JG, Tepper CG, et al. Syngeneic Mouse Mammary Carcinoma Cell Lines: Two Closely Related
Cell Lines With Divergent Metastatic Behavior. Clin Exp Metastasis (2005) 22 (1):47-59. doi: 10.1007/s10585-005-2908-5

33. Rutkowski MR, Allegrezza MJ, Svoronos N, Tesone AJ, Stephen TL, PeralesPuchalt A, et al. Initiation of Metastatic Breast Carcinoma by Targeting of the Ductal Epithelium With Adenovirus-Cre: A Novel Transgenic Mouse Model of Breast Cancer. J Vis Exp (2014) (85). doi: 10.3791/51171

34. Chen BJ, Cui X, Sempowski GD, Liu C, Chao NJ. Transfer of Allogeneic CD62L- Memory T Cells Without Graft-Versus-Host Disease. Blood (2004) 103(4):1534-41. doi: 10.1182/blood-2003-08-2987

35. Youn JI, Nagaraj S, Collazo M, Gabrilovich DI. Subsets of Myeloid-Derived Suppressor Cells in Tumor-Bearing Mice. J Immunol (2008) 181(8):5791-802. doi: 10.4049/jimmunol.181.8.5791

36. Moore MW, Carbone FR, Bevan MJ. Introduction of Soluble Protein Into the Class I Pathway of Antigen Processing and Presentation. Cell (1988) 54 (6):777-85. doi: 10.1016/s0092-8674(88)91043-4

37. Franklin RA, Liao W, Sarkar A, Kim MV, Bivona MR, Liu K, et al. The Cellular and Molecular Origin of Tumor-Associated Macrophages. Science (2014) 344(6186):921-5. doi: 10.1126/science.1252510

38. Tokumitsu H, Inuzuka H, Ishikawa Y, Ikeda M, Saji I, Kobayashi R. STO609, a Specific Inhibitor of the $\mathrm{Ca}(2+) /$ calmodulin-Dependent Protein Kinase Kinase. J Biol Chem (2002) 277(18):15813-8. doi: 10.1074/ jbc.M201075200

39. Barnes TA, Amir E. HYPE or HOPE: The Prognostic Value of Infiltrating Immune Cells in Cancer. Br J Cancer (2017) 117(4):451-60. doi: 10.1038/ bjc. 2017.220

40. Mondanelli G, Volpi C. Differentiation of Myeloid-Derived Suppressor Cells From Murine Bone Marrow and Their Co-Culture With Splenic Dendritic Cells. Bio Protoc (2017) 7(18). doi: 10.21769/BioProtoc.2558

41. Eckert I, Ribechini E, Lutz MB. In Vitro Generation of Murine MyeloidDerived Suppressor Cells, Analysis of Markers, Developmental Commitment, and Function. Methods Mol Biol (2021) 2236:99-114. doi: 10.1007/978-10716-1060-2_10

42. Nakamura K, Kassem S, Cleynen A, Chrétien ML, Guillerey C, Putz EM, et al. Dysregulated IL-18 Is a Key Driver of Immunosuppression and a Possible Therapeutic Target in the Multiple Myeloma Microenvironment. Cancer Cell (2018) 33(4):634-48.e5. doi: 10.1016/j.ccell.2018.02.007

43. Lim HX, Hong HJ, Cho D, Kim TS. IL-18 Enhances Immunosuppressive Responses by Promoting Differentiation Into Monocytic Myeloid-Derived Suppressor Cells. J Immunol (2014) 193(11):5453-60. doi: 10.4049/ jimmunol.1401282

44. Redza-Dutordoir M, Averill-Bates DA. Activation of Apoptosis Signalling Pathways by Reactive Oxygen Species. Biochim Biophys Acta (2016) 1863 (12):2977-92. doi: 10.1016/j.bbamcr.2016.09.012

45. Nugud A, Sandeep D, El-Serafi AT. Two Faces of the Coin: Minireview for Dissecting the Role of Reactive Oxygen Species in Stem Cell Potency and Lineage Commitment. J Adv Res (2018) 14:73-9. doi: 10.1016/ j.jare.2018.05.012

46. Kusmartsev S, Gabrilovich DI. Inhibition of Myeloid Cell Differentiation in Cancer: The Role of Reactive Oxygen Species. J Leukoc Biol (2003) 74(2):18696. doi: $10.1189 / \mathrm{jlb} .0103010$

47. Beury DW, Carter KA, Nelson C, Sinha P, Hanson E, Nyandjo M, et al. Myeloid-Derived Suppressor Cell Survival and Function Are Regulated by the Transcription Factor Nrf2. J Immunol (2016) 196(8):3470-8. doi: 10.4049/ jimmunol.1501785

48. Jian SL, Chen WW, Su YC, Su YW, Chuang TH, Hsu SC, et al. Glycolysis Regulates the Expansion of Myeloid-Derived Suppressor Cells in TumorBearing Hosts Through Prevention of ROS-Mediated Apoptosis. Cell Death Dis (2017) 8(5):e2779. doi: 10.1038/cddis.2017.192

49. Görlach A, Bertram K, Hudecova S, Krizanova O. Calcium and ROS: A Mutual Interplay. Redox Biol (2015) 6:260-71. doi: 10.1016/ j.redox.2015.08.010

50. Madreiter-Sokolowski CT, Thomas C, Ristow M. Interrelation Between ROS and Ca. Redox Biol (2020) 36:101678. doi: 10.1016/j.redox.2020.101678

51. Sundararaman A, Amirtham U, Rangarajan A. Calcium-Oxidant Signaling Network Regulates AMP-Activated Protein Kinase (AMPK) Activation Upon Matrix Deprivation. J Biol Chem (2016) 291(28):14410-29. doi: 10.1074/ jbc.M116.731257 
52. Wang S, Yi X, Wu Z, Guo S, Dai W, Wang H, et al. CAMKK2 Defines Ferroptosis Sensitivity of Melanoma Cells by Regulating AMPK-Nrf2 Pathway. J Invest Dermatol (2021). doi: 10.1016/j.jid.2021.05.025

53. Festjens N, Kalai M, Smet J, Meeus A, Van Coster R, Saelens X, et al. Butylated Hydroxyanisole Is More Than a Reactive Oxygen Species Scavenger. Cell Death Differ (2006) 13(1):166-9. doi: 10.1038/sj.cdd.4401746

54. Dikalova AE, Bikineyeva AT, Budzyn K, Nazarewicz RR, McCann L, Lewis W, et al. Therapeutic Targeting of Mitochondrial Superoxide in Hypertension. Circ Res (2010) 107(1):106-16. doi: 10.1161/CIRCRESAHA. 109.214601

55. Hamanaka RB, Chandel NS. Mitochondrial Reactive Oxygen Species Regulate Cellular Signaling and Dictate Biological Outcomes. Trends Biochem Sci (2010) 35(9):505-13. doi: 10.1016/j.tibs.2010.04.002

56. Scialò F, Fernández-Ayala DJ, Sanz A. Role of Mitochondrial Reverse Electron Transport in ROS Signaling: Potential Roles in Health and Disease. Front Physiol (2017) 8:428. doi: 10.3389/fphys.2017.00428

57. Rabinovitch RC, Samborska B, Faubert B, Ma EH, Gravel SP, Andrzejewski S, et al. AMPK Maintains Cellular Metabolic Homeostasis Through Regulation of Mitochondrial Reactive Oxygen Species. Cell Rep (2017) 21(1):1-9. doi: 10.1016/j.celrep.2017.09.026

58. Herzig S, Shaw RJ. AMPK: Guardian of Metabolism and Mitochondrial Homeostasis. Nat Rev Mol Cell Biol (2018) 19(2):121-35. doi: 10.1038/ nrm. 2017.95

59. Hinchy EC, Gruszczyk AV, Willows R, Navaratnam N, Hall AR, Bates G, et al. Mitochondria-Derived ROS Activate AMP-Activated Protein Kinase (AMPK) Indirectly. J Biol Chem (2018) 293(44):17208-17. doi: 10.1074/jbc. RA118.002579

60. Kortylewski M, Kujawski M, Wang T, Wei S, Zhang S, Pilon-Thomas S, et al. Inhibiting Stat3 Signaling in the Hematopoietic System Elicits Multicomponent Antitumor Immunity. Nat Med (2005) 11(12):1314-21. doi: $10.1038 / \mathrm{nm} 1325$

61. Abad C, Nobuta H, Li J, Kasai A, Yong WH, Waschek JA. Targeted STAT3 Disruption in Myeloid Cells Alters Immunosuppressor Cell Abundance in a Murine Model of Spontaneous Medulloblastoma. J Leukoc Biol (2014) 95 (2):357-67. doi: 10.1189/jlb.1012531
62. Sullivan JE, Brocklehurst KJ, Marley AE, Carey F, Carling D, Beri RK. Inhibition of Lipolysis and Lipogenesis in Isolated Rat Adipocytes With AICAR, a Cell-Permeable Activator of AMP-Activated Protein Kinase. FEBS Lett (1994) 353(1):33-6. doi: 10.1016/0014-5793(94)01006-4

63. Condamine T, Gabrilovich DI. Molecular Mechanisms Regulating MyeloidDerived Suppressor Cell Differentiation and Function. Trends Immunol (2011) 32(1):19-25. doi: 10.1016/j.it.2010.10.002

64. Ugel S, De Sanctis F, Mandruzzato S, Bronte V. Tumor-Induced Myeloid Deviation: When Myeloid-Derived Suppressor Cells Meet Tumor-Associated Macrophages. J Clin Invest (2015) 125(9):3365-76. doi: 10.1172/JCI80006

65. Hayes JD, Dinkova-Kostova AT. The Nrf2 Regulatory Network Provides an Interface Between Redox and Intermediary Metabolism. Trends Biochem Sci (2014) 39(4):199-218. doi: 10.1016/j.tibs.2014.02.002

66. Kovac S, Angelova PR, Holmström KM, Zhang Y, Dinkova-Kostova AT, Abramov AY. Nrf2 Regulates ROS Production by Mitochondria and NADPH Oxidase. Biochim Biophys Acta (2015) 1850(4):794-801. doi: 10.1016/ j.bbagen.2014.11.021

Conflict of Interest: The authors declare that the research was conducted in the absence of any commercial or financial relationships that could be construed as a potential conflict of interest.

Publisher's Note: All claims expressed in this article are solely those of the authors and do not necessarily represent those of their affiliated organizations, or those of the publisher, the editors and the reviewers. Any product that may be evaluated in this article, or claim that may be made by its manufacturer, is not guaranteed or endorsed by the publisher.

Copyright (c) 2021 Huang, Liu, Luz, Berrong, Meyer, Zou, Swann, Sundaramoorthy, Kang, Jauhari, Lento, Chao and Racioppi. This is an open-access article distributed under the terms of the Creative Commons Attribution License (CC BY). The use, distribution or reproduction in other forums is permitted, provided the original author(s) and the copyright owner(s) are credited and that the original publication in this journal is cited, in accordance with accepted academic practice. No use, distribution or reproduction is permitted which does not comply with these terms. 